\title{
Polarization fluctuations in vertical-cavity semiconductor lasers
}

\author{
M. P. van Exter, M. B. Willemsen, and J. P. Woerdman \\ Huygens Laboratory, Leiden University, P.O. Box 9504, 2300 RA Leiden, The Netherlands
}

(Received 1 May 1998; revised manuscript received 24 July 1988)

\begin{abstract}
We report, theoretically and experimentally, how polarization fluctuations in vertical-cavity semiconductor lasers are affected by optical anisotropies. We develop a spin-eliminated (class A) description of laser polarization and show how the various model parameters can be extracted from the experimental data. In practice, the linear anisotropies are often much stronger than the nonlinear anisotropies, so that the polarization modes defined by the linear anisotropies form a useful basis. For this case we derive a one-dimensional model for polarization noise, with simple expressions for the relative strength of the polarization fluctuations and the rate of polarization switches. For the other, more extreme, case where the nonlinear anisotropies are as strong (or even stronger) than the linear anisotropies, the spin-eliminated description remains valid. However, in this case the concept of polarization modes is shown to lose its meaning, as a strong four-wave-mixing peak appears in the optical spectrum and polarization fluctuations become highly nonuniform. [S1050-2947(98)06311-2]
\end{abstract}

PACS number(s): 42.55.Px

\section{INTRODUCTION}

Polarization fluctuations are present in all lasers, but are exceptionally strong in semiconductor vertical-cavity surface-emitting lasers (VCSELs). The reason for this is twofold. On the one hand, the spontaneous emission noise, which drives the polarization fluctuations, is relatively strong due to the limited size of the device. This is true for any semiconductor laser and leads, among others, to a relatively large quantum-limited laser linewidth [1]. On the other hand, the deterministic forces, being the optical anisotropies in the device, are relatively small due to the nominal cylindrical symmetry of a VCSEL. The combination of strong stochastic noise and weak restoring forces creates relatively large polarization fluctuations. A proper understanding of these fluctuations is clearly important from a practical point of view; in any application of VCSELs polarization noise will be converted into intensity noise by the (unavoidable) polarization dependence of a practical detection system. Alternatively, and this is the emphasis of the present paper, a study of these fluctuations constitutes a very useful tool to unravel the various anisotropies and other laser parameters of practical VCSELs. A preliminary report of our study has appeared recently [2].

Until recently, it was difficult to compare theory and experiments on VCSEL polarization. The "standard" theoretical model for the polarization of a quantum-well VCSEL is the "split-inversion model," developed by San Miguel, Feng, and Moloney [3]. In this model the conduction and heavy-hole valence band are treated as four discrete levels, with $M= \pm \frac{1}{2}$ and $M= \pm \frac{3}{2}$, respectively, and the inversion is split into two transitions $\left(M=\frac{1}{2} \leftrightarrow \frac{3}{2}\right.$ and $\left.M=-\frac{1}{2} \leftrightarrow-\frac{3}{2}\right)$, each interacting with circularly polarized light of a specific handedness. An important parameter in this model is $\Gamma$, which describes the spin-flip relaxation between the two spin inversions (normalized to the inversion decay rate). Unfortunately, the split-inversion model is rather complicated, as the dynamics of two population inversions has to be accounted for, so that analytic approaches are very difficult. Numerical studies have concentrated on the issue of polarization stabil- ity, depicted in so-called stability diagrams, and more specifically on polarization switching and bistability [4-6]. Although polarization switching has been observed in several experiments, a quantitative comparison with theory proved to be difficult, due to the numerical methodology and due to the fact that only limited information could be extracted from the experiments reported so far $[7,8]$. Also, alternative explanations for polarization switches seemed equally likely [7].

Other experimental studies involved the optical spectra of light emitted by VCSELs [9]. In general, these spectra consist of two (Lorentzian-shaped) components, a strong "lasing mode" and a weak "nonlasing mode" with orthogonal polarization, the two components being related to the two VCSEL polarizations. The differences in center frequency and spectral width between these two components could be almost completely attributed to linear anisotropies; only small deviations between experiment and a linear "coupledmode" model hinted at more complicated population dynamics [9]. In practice, nonlinear anisotropies were thus found to be relatively small, corresponding to a large value of $\Gamma$.

A reconciliation between experiment and theory came with a simplified theoretical description, which was concurrently developed by several authors $[6,10,11]$, in which the spin inversion was adiabatically eliminated from the laser rate equations. This leads to a first-order separation of the polarization and intensity/inversion dynamics, so that the polarization dynamics of a VCSEL is that of a class A laser, although the intensity dynamics is still that of a class B laser (with relaxation oscillations). In this model, the effect of the eliminated spin inversion is still contained in the rate equations for the optical field, namely, as a nonlinear anisotropy or polarization-dependent optical saturation, the saturation power for linearly polarized light being (slightly) larger than for circularly polarized light.

As a next step the rate equations are generally linearized around steady state. The simplicity of the linearized spineliminated model allows for many analytic expressions; the model yields, among others, expressions for a nonlinear redshift and for excess damping of the nonlasing mode as com- 
pared to the lasing mode $[6,10,11]$. More recent predictions [11] concern the appearance of a third (four-wave-mixing) peak in the optical spectrum and asymmetries in the polarization-resolved intensity noise (see below).

Theoretically, this paper constitutes an extension of the work on the spin-eliminated model reported in [6] and [11]. We will put special emphasis on the role of noise. The key issue is not so much whether the lasing polarization is stable, but rather how stable it is, what the stability eigenvalues are, and how much the polarization still fluctuates around its equilibrium value. We hereby derive many useful expressions for VCSEL polarization noise that allow for easy comparison with experiment. As a further extension, we will go beyond the linearized theory, concentrating on the practical case that linear birefringence is the dominant anisotropy, and study the dynamics of polarization switching in VCSELs. Experimentally, we report a multitude of data on VCSEL polarization noise, extending the work reported in [2]. By analyzing the measured polarization fluctuations, which can be exceptionally strong in VCSELs, we extract a series of VCSEL parameters, with emphasis on the various optical anisotropies.

We focus on three experimental tools to study the polarization fluctuations. The first tool is a measurement of the polarization-resolved optical spectrum, where polarization fluctuations show up in the form of additional spectral peaks with a polarization different from that of the lasing peak. The second tool is a measurement of the polarization-resolved intensity noise, a polarization-type of homodyne detection, suggested by Hofmann and Hess [11] and first demonstrated in [2], in which the intensity noise, after polarization projection of the VCSEL output, is frequency analyzed. We will show how this technique provides information on the polarization fluctuations. As a third tool we employ a timedomain study of the polarization-resolved intensity.

In Sec. II we will briefly review the adiabatic model for the polarization dynamics of VCSELs. In doing so we will generalize the earlier theory to the case of nonaligned birefringence and dichroism. After discussing the various parameters in the problem, we will show how their magnitude can be determined from experimental data. To facilitate the comparison between theory and experiment, Secs. III and IV present several useful expressions for the polarizationresolved optical spectrum, and the polarization-resolved intensity noise, respectively.

In Sec. V we isolate the case where the linear birefringence dominates over all other anisotropies; this is the case encountered for almost any practical VCSEL. We will show how in this case the adiabatic description in terms of two polarization variables can be reduced even further, to a simple one-dimensional description, with appealing expressions for the relative strength of the polarization fluctuations and the hopping rate in case of polarization switching.

In Secs. VI-X we present and analyze our experimental data, organized via the three basic techniques that we use. In Sec. VI we discuss the experimental setup, in Sec. VII the polarization-resolved optical spectra, in Sec. VIII the polarization-resolved intensity noise, and in Sec. IX the polarization switches that occur in some VCSELs. In Sec. X we will discuss results for VCSELs with a different design,
TABLE I. Important parameters and variables, together with their symbol and units.

\begin{tabular}{lll}
\hline \hline Parameter or variable & \multicolumn{1}{c}{ Symbol } & Units \\
\hline Linear birefringence & $\omega_{\text {lin }}$ & $\mathrm{ns}^{-1}$ \\
Linear dichroism & $\gamma_{\text {lin }}$ & $\mathrm{ns}^{-1}$ \\
Projected linear dichroism & $\gamma_{\|}=\gamma_{\text {lin }} \cos 2 \beta$ & $\mathrm{ns}^{-1}$ \\
Angle between lin. birefringence & $\beta$ & $\mathrm{rad}^{\prime}$ \\
$\quad$ \& lin. dichroism & & \\
Nonlinear birefringence & $\omega_{\mathrm{non}}=\alpha \gamma_{\mathrm{non}}$ & $\mathrm{ns}^{-1}$ \\
Nonlinear dichroism & $\gamma_{\mathrm{non}}$ & $\mathrm{ns}^{-1}$ \\
Henry's phase-amplitude coupling factor & $\alpha$ & \\
Effective birefringence & $\omega_{0}=2 \pi \nu_{0}$ & $\mathrm{~ns}^{-1}$ \\
Effective dichroism & $\gamma_{0}=\gamma_{\|}+\gamma_{\mathrm{non}}$ & $\mathrm{ns}^{-1}$ \\
Cavity loss rate & $\kappa$ & $\mathrm{ns}^{-1}$ \\
$\quad$ (of intracavity optical field) & & \\
Loss rate of average inversion & $\gamma$ & $\mathrm{ns}^{-1}$ \\
Loss rate of difference inversion & $\gamma_{\mathrm{s}}$ & $\mathrm{ns}^{-1}$ \\
Normalized spin decay rate & $\Gamma=\gamma_{s} / \gamma$ & \\
Noise strength & $D=n_{\mathrm{sp}} \kappa / S$ & $\mathrm{~ns}^{-1}$ \\
Spontaneous emission factor & $n_{\mathrm{sp}}$ & \\
Number of photons in & $S$ & \\
$\quad$ fundamental cavity mode & & \\
Intracavity intensity & $\mathrm{I}$ & \\
$\quad$ (normalized to saturation) & & $\mathrm{rad}$ \\
Polarization orientation angle & $\phi$ & $\mathrm{rad}$ \\
Polarization ellipticity angle & $\chi$ & \\
Rotationally averaged polarization angle & $\varphi$ & \\
\hline \hline
\end{tabular}

whereas Sec. XI summarizes the results and gives an overall conclusion.

\section{ADIABATIC DESCRIPTION OF POLARIZATION FLUCTUATIONS}

Since we do not want to copy the derivation of the starting equations we refer to [3-5] for the standard splitinversion model and to $[6,11]$ for the spin-eliminated version of that model. The validity condition for the adiabatic elimination has been thoroughly discussed in [6]: the polarization of the optical field should vary slowly as compared to the medium response to polarization changes. This means that (i) the optical anisotropies should not be too large, as these set the time scale of polarization changes, and (ii) the normalized spin-decay rate $\Gamma$ should be large enough, as this sets the time scale of the medium response.

We will spend some effort in defining the parameters and variables of the problem (see Table I), as the literature is far from uniform in this respect $[3-6,11]$. As variables we use the average inversion $N$ (normalized to the threshold inversion) and the optical field vector $\operatorname{Re}\left[\vec{E} e^{-i \omega_{l} t}\right]$. The latter can be separated into two complex field components $E_{x}$ and $E_{y}$ (or $E_{+}$and $E_{-}$). This is, however, somewhat inconvenient as nonlinear anisotropies create correlations between the fluctuations in these components. We will therefore anticipate the expected separation between the polarization and intensity/inversion dynamics, and describe the optical field vector with four real-valued variables, instead. The first variable is a common phase factor (the phase of the laser field 
$\varphi_{l}$ ), which exhibits a diffusive evolution that has no consequences for the other dynamics. The other variables are the optical intensity $I=|\vec{E}|^{2}$, and two Poincaré angles $\phi$ and $\chi$ that characterize the optical polarization [12], where $\phi(0$ $\leqslant \phi \leqslant \pi)$ is the direction of the polarization ellipse and $\chi$ $(-\pi / 4 \leqslant \chi \leqslant \pi / 4)$ is the ellipticity angle. For practical VCSELs the output polarization is practically always close to linear, in a direction that we can define to be the $x$ axis. Linearization around this point yields

$$
\vec{E} \approx\left[\vec{e}_{x}-(\phi+i \chi) \vec{e}_{y}\right]|\vec{E}| e^{-i \varphi_{l}} .
$$

The original split-inversion model contains three decay rates: the decay rate $\kappa$ for the optical field, the decay rate $\gamma$ for the average inversion $\left(N_{+}+N_{-}\right) / 2$, and the decay rate $\gamma_{s}$ for the difference inversion $\left(N_{+}-N_{-}\right) / 2$, where $\Gamma=\gamma_{s} / \gamma$. Adiabatic elimination of the difference inversion clearly demonstrates and isolates the polarization dependence of the optical saturation. The magnitude of the corresponding nonlinear anisotropies is $\gamma_{\text {non }}=\kappa I / \Gamma$ for the nonlinear dichroism (absorptive saturation) and $\omega_{\text {non }}=\alpha \gamma_{\text {non }}$ for the nonlinear birefringence (dispersive saturation), where $\alpha$ is Henry's phase-amplitude coupling factor [1]. These nonlinear anisotropies are proportional to the intracavity intensity $I$, which has been normalized with respect to the saturation intensity and which for an ideal four-level laser is thus equal to the normalized pump parameter $\mu$ [6].

The rotational polarization symmetry of a laser is generally broken by linear anistropies, i.e., anisotropies that are independent of laser power. In the absence of a magnetic field there can be only two of these: a birefringence $\omega_{\text {lin }}$ and a dichroism $\gamma_{\operatorname{lin}}[5,13]$. As these anisotropies have a directionality, we also need the angle $\beta$ between the axes of linear dichroism and linear birefringence. A summary of the parameters that we use is given in Table I. For easy comparison with the literature we note that our symbols $\omega_{\operatorname{lin}}$ and $\gamma_{\text {lin }}$, for the linear birefringence and linear dichroism, correspond to $2 \sigma$ and $-2 \epsilon$ in $[5,6,14]$, to $-2 \gamma_{p}$ and $-2 \gamma_{a}$ in [4], and to $\Omega$ and $s$ in [11], respectively. Furthermore, the nonlinear dichroism $\gamma_{\text {non }}$ is denoted as $\kappa I / \Gamma$ in $[5,14]$, as $\kappa \mu / \Gamma$ in [6], as $\kappa(\mu-1) / \Gamma$ in [4], and as $\chi n / 2$ in [11]. Note that the linear birefringence and linear dichroism both have a sign, being positive when the lasing mode has the highest frequency and highest linear gain, respectively.

In earlier work the linear birefringence and linear dichroism were often assumed to be aligned, resulting in VCSEL eigenmodes that are linearly polarized along the common axes of birefringence and dichroism. We now generalize this approach, allowing the axes of linear birefringence and linear dichroism to make an arbitrary angle $\beta$. In the Appendix the full expressions for this general case, from [5,13,14], are rewritten into the following linearized polarization rate equations:

$$
\frac{d}{d t}\left(\begin{array}{c}
\phi-\phi_{\mathrm{ss}} \\
\chi-\chi_{\mathrm{ss}}
\end{array}\right)=\left(\begin{array}{cc}
-\gamma_{\|} & -\omega_{\mathrm{lin}}-2 \alpha \gamma_{\mathrm{non}} \\
\omega_{\mathrm{lin}} & -\gamma_{\|}-2 \gamma_{\mathrm{non}}
\end{array}\right)\left(\begin{array}{c}
\phi-\phi_{\mathrm{ss}} \\
\chi-\chi_{\mathrm{ss}}
\end{array}\right)+\left(\begin{array}{c}
f_{\phi} \\
f_{\chi}
\end{array}\right),
$$

where $\gamma_{\|}=\gamma_{\text {lin }} \cos 2 \beta, \phi_{\mathrm{ss}}$ and $\chi_{\mathrm{ss}}$ are steady-state angles, and $f_{\phi}$ and $f_{\chi}$ are Langevin noise sources. Misalignment is thus found to result in two changes: (i) the steady-state po- larization ceases to be linear and obtains an average ellipticity $\chi_{\mathrm{ss}}\left[\right.$ where we consider only $\chi_{\mathrm{ss}} \ll 1$, see Eq. (A2a)], and (ii) the polarization dynamics is now determined by the projected linear dichroism $\gamma_{\|}=\gamma_{\operatorname{lin}} \cos 2 \beta$.

For completeness we note that, in some aspects, the validity range of Eq. (2) surpasses that of the underlying splitinversion model. Namely, through adiabatic elimination we have reduced our description to a general third-order Lamb theory for the laser polarization, which is valid for any class A laser with rotational symmetry [6]. In this sense Eq. (2) is quite general; it is only the interpretation of the nonlinear anisotropies $\gamma_{\text {non }}$ and $\omega_{\text {non }}$, as $\gamma_{\text {non }}=\kappa I / \Gamma$ and $\omega_{\text {non }}$ $=\alpha \gamma_{\text {non }}$, that is specific for the split-inversion model.

The eigenvalues of the above equation [Eq. (2)] are $\lambda=$ $-\gamma_{0} \pm i \omega_{0}$, with

$$
\begin{gathered}
\gamma_{0}=\gamma_{\|}+\gamma_{\text {non }}, \\
\omega_{0}=\sqrt{\omega_{\operatorname{lin}}^{2}+2 \omega_{\operatorname{lin}} \alpha \gamma_{\text {non }}-\gamma_{\text {non }}^{2}} \\
=\sqrt{\left(\omega_{\operatorname{lin}}+\alpha \gamma_{\text {non }}\right)^{2}-\left(\alpha^{2}+1\right) \gamma_{\text {non }}^{2}},
\end{gathered}
$$

where $\omega_{0}$ and $\gamma_{0}$ contain the combined action of linear and nonlinear effects and will thus be called the effective birefringence and effective dichroism, respectively, and where the nonlinear terms corresponds to a "spectral redshift" and "excess broadening" of the nonlasing peak as compared to the lasing peak $[6,10,11]$. The corresponding eigenvectors are

$$
\left(\begin{array}{c}
\omega_{\text {lin }}+2 \alpha \gamma_{\text {non }} \\
\mp i \omega_{0}+\gamma_{\text {non }}
\end{array}\right) \approx \omega_{0}\left(\begin{array}{c}
1 \\
\mp i
\end{array}\right)+\left(\begin{array}{c}
\alpha \gamma_{\text {non }} \\
\gamma_{\text {non }}
\end{array}\right)
$$

where the approximate expression is valid for $\omega_{0}$ $\gg \sqrt{\alpha^{2}+1} \gamma_{\text {non }}$.

The main reason for writing down the above eigenvectors [Eq. (4)] is that these already show the intrinsic polarization dynamics, i.e., the response to a perturbation without noise. In the absence of nonlinear anisotropies, i.e., for $\gamma_{\text {non }}=0$, the dynamics is extremely simple: on the Poincare sphere the polarization $(\phi, \chi)$ will evolve along a spiral-like curve towards steady state. In terms of optical amplitudes this means that there is a (steady-state) lasing mode and an (orthogonally polarized) nonlasing mode that gradually decays to zero. The rotation on the Poincare sphere is counterclockwise for the case $\omega_{0}>0$, where the dominant $x$-polarized mode has the highest frequency.

In the presence of nonlinear anisotropies the situation becomes more interesting. Equation (4) shows that the amplitudes in the $\phi$ and $\chi$ directions will then be different, so that the evolution is now along an elliptical spiral-like trajectory. As a consequence, fluctuations in the laser's polarization direction $\phi$ are expected to have a different magnitude than fluctuations in the ellipticity $\chi$. As another consequence a third peak is expected to appear in the optical spectrum. This is because the mentioned trajectory can be decomposed in a clockwise and counterclockwise circular trajectory, which correspond to spectral peaks on the high- and low-frequency sides of the lasing peak, respectively [2,11]. The approximate amplitude of these components can be easily found from Eq. (4). 
Equation (2) shows how polarization fluctuations result from a balance between the stochastic driving force of polarization noise and the damping and spectral deformation caused by the various anisotropies. The polarization noise is a manifestation of the quantum noise that results from the discrete character of photons and carriers. For practical VCSELs $\kappa / \gamma \gg 1$, so that photon noise dominates, as the average number of inverted carrier states is much larger than the average photon number. As photon noise originates from random spontaneous emission of photons with arbitrary phase and arbitrary polarization $\left(N_{+} \approx N_{-}\right)$, the complex noise vector $\vec{f}(t)$ comprises four independent real-valued numbers, that can be divided into phase noise, intensity noise, and two forms of polarization noise. Phase and amplitude noise are best known as they also occur in the singlemode (scalar) problem. The two polarization components are similar uncorrelated real-valued Langevin noise sources of identical strength, which satisfy

$$
\begin{gathered}
\left\langle f_{\chi}\left(t_{1}\right) f_{\chi}\left(t_{2}\right)\right\rangle=\left\langle f_{\phi}\left(t_{1}\right) f_{\phi}\left(t_{2}\right)\right\rangle=D \delta\left(t_{1}-t_{2}\right), \\
\left\langle\left|f_{\chi}(\omega)\right|^{2}\right\rangle=\left\langle\left|f_{\phi}(\omega)\right|^{2}\right\rangle=D=n_{\mathrm{sp}} \kappa / S,
\end{gathered}
$$

where the noise strength, or diffusion rate $D$, is inversely proportional to the photon number $S$ and proportional to the product of cavity loss rate $\kappa$ and spontaneous emission factor $n_{\mathrm{sp}}\left(n_{\mathrm{sp}} \geqslant 1\right.$ results from incomplete inversion as determined by the finite temperature, which smoothens the sharpness of the Fermi-Dirac distribution) [15].

One way to solve the polarization rate equations (2) is via Green functions that are based on the eigenvectors of Eq. (4); this was done in [11]. An easier way is to apply a Fourier transformation and solve the equations in the frequency domain, to obtain

$$
\begin{gathered}
\phi(\omega)=\frac{\left(i \omega-\gamma_{\|}-2 \gamma_{\mathrm{non}}\right) f_{\phi}(\omega)+\left(\omega_{\mathrm{lin}}+2 \alpha \gamma_{\mathrm{non}}\right) f_{\chi}(\omega)}{\left(\omega-\omega_{0}-i \gamma_{0}\right)\left(\omega+\omega_{0}-i \gamma_{0}\right)}, \\
\chi(\omega)=\frac{-\omega_{\operatorname{lin}} f_{\phi}(\omega)+\left(i \omega-\gamma_{\|}\right) f_{\chi}(\omega)}{\left(\omega-\omega_{0}-i \gamma_{0}\right)\left(\omega+\omega_{0}-i \gamma_{0}\right)} .
\end{gathered}
$$

By combining these equations with the expressions for the polarization noise [Eqs. (5)] it is relatively straightforward to calculate the experimentally accessible polarization-resolved optical spectra and intensity noise. This will be done in the next section.

For completeness we note that the simplicity of the above results is due to the fact that, after spin elimination, the polarization dynamics $(\phi, \chi)$ is separated almost completely from the other dynamics, namely, that of the intensity $I$, average inversion $N$, and optical phase $\varphi_{l}$. The only coupling is via the intensity dependence of $\gamma_{\text {non }}$ and this coupling disappears when the intensity is reasonably constant, i.e., when fluctuations are limited or at frequencies very different from those of the polarization dynamics, so that one can substitute the average intensity. As a result the polarization dynamics of a VCSEL can be that of a class A laser, whereas the (relatively weak) intensity fluctuations that are still present can be those of a class B laser that exhibits relaxation oscillations $[15,16]$.

\section{POLARIZATION-RESOLVED OPTICAL SPECTRA}

In this section we will calculate the optical spectrum $|E(\omega)|^{2}$ of the VCSEL light, as measured after polarization projection. In the linearized description, i.e., for $\phi, \chi \ll 1$, the projection onto the dominant polarization depends only on the dynamics of the optical phase and intensity (see below). On the other hand, if we block this light and project onto the orthogonal polarization, we obtain different information, namely, on the polarization dynamics. The optical spectrum thus observed is the Fourier transformation of $E_{y}(t) \approx$ $-[\phi(t)+i \chi(t)] E(t) \exp \left[-i \varphi_{l}(t)\right], \quad$ where $\quad E(t) \equiv|\vec{E}(t)|$ $\approx\left|E_{x}(t)\right|$. For convenience, we will first assume the optical field and optical phase to be constant at $E(t) \exp \left[-i \varphi_{l}(t)\right]$ $=E_{0}$; later we will remove this restriction. In this practical case, the $y$-polarized spectrum is dominated by the polarization dynamics, so that

$$
\left\langle\left|E_{y}(\omega)\right|^{2}\right\rangle \approx E_{0}^{2}\left\langle|\phi(\omega)+i \chi(\omega)|^{2}\right\rangle=D E_{0}^{2} \frac{\left(\omega-\omega_{\operatorname{lin}}\right)^{2}+\left(\omega-\omega_{\operatorname{lin}}-2 \alpha \gamma_{\text {non }}\right)^{2}+\gamma_{\|}^{2}+\left(\gamma_{\|}+2 \gamma_{\text {non }}\right)^{2}}{\left(\omega^{2}-\omega_{0}^{2}-\gamma_{0}^{2}\right)^{2}+4 \gamma_{0}^{2} \omega^{2}}
$$

This optical spectrum generally consists of two peaks: a strong peak at $\omega \approx-\omega_{0}$, which corresponds to the "nonlasing mode" in the coupled-mode description [9], and a (much weaker) peak at $\omega \approx \omega_{0}$, which is produced in a polarization type of four-wave mixing (FWM) between the $y$-polarized peak at $\omega \approx-\omega_{0}$ and the dominant $x$-polarized peak at $\omega$ $=0$ [2]. The $y$-polarized spectrum can be approximated as the sum of two Lorentzian curves with the same width when $\omega_{0} \gg \gamma_{0}$. The position and width (HWHM, half width at half maximum) of the two peaks yield the effective birefringence $\omega_{0}$ and the effective dichroism $\gamma_{0}$, respectively. The intensity of the FWM peak, relative to that of the nonlasing peak, can then be used to estimate the combined (i.e., dispersive and absorptive) nonlinear anisotropy $\left(\alpha^{2}+1\right) \gamma_{\text {non }}^{2}$ via

$$
\frac{\left\langle\left|E_{y}\left(\omega_{0}\right)\right|^{2}\right\rangle}{\left\langle\left|E_{y}\left(-\omega_{0}\right)\right|^{2}\right\rangle} \approx \frac{\left(\alpha^{2}+1\right) \gamma_{\text {non }}^{2}}{4 \omega_{0}^{2}}+\frac{\gamma_{0}^{2}}{4 \omega_{0}^{2}}
$$

where the second term results from the Lorentzian wing of the nonlasing peak at the position of the FWM peak. Note that a decomposition of the eigenvectors [Eq. (4)] in their $\mathrm{cw}$ and ccw components gives the same approximate result. 
It is relatively easy to go beyond the approximation of "constant $E(t)$ and $\varphi_{l}(t)$ " by noting that the polarizationresolved optical field is the product of the field $E(t) \exp \left[-i \varphi_{l}(t)\right]$ times a function of $(\phi, \chi)$. As a result, in the general case the polarization-resolved spectrum equals the convolution of the ideal spectrum [Eq. (7)] with the spectrum $\left|E(\omega)^{2}\right| \approx\left|E_{x}(\omega)\right|^{2}$, as measured for projection onto the dominant polarization. The shape of the latter is similar to that of "edge-emitting" lasers: it has a finite (SchawlowTownes) laser linewidth $\gamma_{\text {lase }}$, due to diffusion of the optical phase, and (generally very weak) sidebands due to relaxation oscillations [16]. After convolution one thus finds that phase diffusion broadens all spectral peaks by an equal amount $\gamma_{\text {lase }}$, being the (HWHM) spectral width of $\left|E_{x}(\omega)\right|^{2}$, but that it does not affect the relative strength of the FWM peak as compared to the nonlasing peak, since these have the same (intrinsic) width (for $\omega_{0} \gg \gamma_{0}$ ).

\section{POLARIZATION-RESOLVED INTENSITY NOISE}

Next we will discuss the polarization-resolved intensity noise. A measurement of this projected noise is extremely simple: the laser light is passed through a rotatable $\lambda / 4$ waveplate and subsequently through a rotatable polarizer, to project $\vec{E}(t)$ onto a selectable polarization state, after which the projected intensity noise is measured. Projection onto the dominant $x$ or orthogonal $y$ polarization yields information about the "polarization-mode partition noise" [17]. The intensity noise in the orthogonal $y$ projection is generally rather small, being second order in $\phi$ and $\chi$ [see Eq. (1)]. A much stronger signal, i.e., first order in $\phi$ and/or $\chi$, is found for projection onto a "mixed" polarization like $x+y$ or $x$ $+i y$. Such a projection constitutes a polarization homodyne detection, because it allows one to observe beats between the $x$-polarized lasing peak and the $y$-polarized nonlasing and FWM peaks [11]. Through these intensity beats, which go unnoticed without projection, one gets a quantitative measure for the polarization fluctuations in the laser.

An appealing picture of the principle behind polarization projection arises when we introduce the Poincaré sphere. On this sphere each polarization state is depicted as a single point, i.e., the normalized Stokes vector $\left(P_{1}, P_{2}, P_{3}\right)$ $\equiv(\cos 2 \chi \cos 2 \phi, \cos 2 \chi \sin 2 \phi, \sin 2 \chi)$, where the equator corresponds to all states of linear polarization, the poles to the two states of circular polarization, and the rest to elliptically polarized light. On the Poincare sphere, the polarization evolution is represented by a time trace and polarization fluctuations by a "noise cloud." Figure 1 sketches how, for dominantly $x$-polarized light, this noise cloud is located in

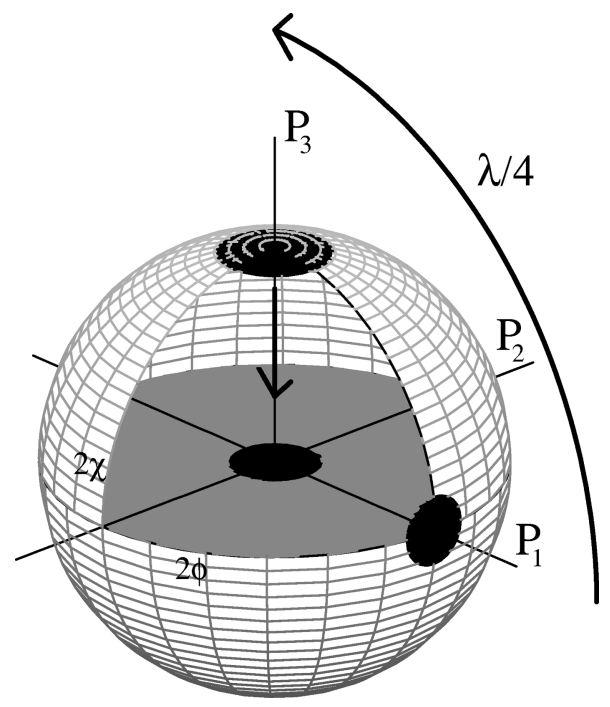

FIG. 1. Principle of noise projection on the Poincare sphere. The polarization fluctuations around the, almost linearly polarized, steady state are represented as a noise cloud around a position close to the equator. Propagation through a $\lambda / 4$ plate and polarizer results in a $90^{\circ}$ rotation towards the north pole and a projection downwards onto an axis, the orientation of which depends on polarizer angle. By projecting onto axis $P_{1}$ or $P_{2}$ we can measure the noise in the Poincaré angles $\chi$ or $\phi$, respectively.

the neighborhood of the equator at $\phi, \chi \ll 1$. When the light is passed through a $\lambda / 4$ plate, with its axes at $45^{\circ}$ with respect to the dominant laser polarization, this noise cloud is rotated by $90^{\circ}$ on the sphere, to end up around the north pole (righthanded circular polarization). The projected intensity behind a consecutive polarizer can now be found graphically by projection of the polarization state onto an axis passing through equator and center of the Poincare sphere, with an orientation that depends on the polarizer angle. When the polarizer axis is aligned with that of the lasing mode one projects onto axis $P_{1}$ in Fig. 1 and measures $I_{\text {project }}(t)=(I / 2)(1+\sin 2 \chi)$ $\approx[I(t) / 2][1+2 \chi(t)]$. When the polarizer axis is aligned under $45^{\circ}$ one projects onto axis $P_{2}$ and measures $I_{\text {project }}(t)$ $\approx[I(t) / 2][1+2 \phi(t)]$. Other orientations give linear combinations of these results.

After this discussion, a calculation of the polarizationresolved intensity noise is straightforward. When the overall intensity is stable enough, the projected noise will be determined by the polarization dynamics only, so that the relative intensity noise, for projection onto the $\phi$ or $\chi$ direction respectively, is given by

$$
\begin{gathered}
\frac{\left\langle\left|\Delta I_{\text {project }}(\omega)\right|^{2}\right\rangle}{\left\langle I_{\text {project }}\right\rangle^{2}}=4\left\langle|\phi(\omega)|^{2}\right\rangle=4 D\left(\frac{\omega^{2}+\left(\omega_{\text {lin }}+2 \alpha \gamma_{\text {non }}\right)^{2}+\left(\gamma_{\|}+2 \gamma_{\text {non }}\right)^{2}}{\left(\omega^{2}-\omega_{0}^{2}-\gamma_{0}^{2}\right)^{2}+4 \gamma_{0}^{2} \omega^{2}}\right), \\
\frac{\left\langle\left|\Delta I_{\text {project }}(\omega)\right|^{2}\right\rangle}{\left\langle I_{\text {project }}\right\rangle^{2}}=4\left\langle|\chi(\omega)|^{2}\right\rangle=4 D\left(\frac{\omega^{2}+\omega_{\text {lin }}^{2}+\gamma_{\|}^{2}}{\left(\omega^{2}-\omega_{0}^{2}-\gamma_{0}^{2}\right)^{2}+4 \gamma_{0}^{2} \omega^{2}}\right) .
\end{gathered}
$$


For the case of relatively large birefringence $\left(\omega_{0}\right.$ $\left.\gg \gamma_{0}, \gamma_{\text {non }}, \alpha \gamma_{\text {non }}\right)$, these projected noise spectra are quite similar, both peaking around $\omega_{0}$ and having a spectral width of $\gamma_{0}$ (HWHM). From fits to these spectra one can directly obtain the effective birefringence $\omega_{0}$ and effective dichroism $\gamma_{0}$, without the experimental complication of a finite laser linewidth $\gamma_{\text {lase }}$ that occurs when analyzing the optical spectra.

Interestingly enough, the above spectra have the same functional form as the relative intensity noise (RIN) spectrum. When the intensity fluctuations are relatively small, so that the intensity rate equation can be linearized, this RIN spectrum is given by

$$
\frac{\left\langle|I(\omega)|^{2}\right\rangle}{I_{0}^{2}}=4 D \frac{\omega^{2}+4 \gamma_{\mathrm{ro}}^{2}}{\left(\omega^{2}-\omega_{\mathrm{ro}}^{2}\right)^{2}+4 \gamma_{\mathrm{ro}}^{2} \omega^{2}},
$$

where $\omega_{\text {ro }}$ and $\gamma_{\text {ro }}$ are the relaxation oscillation frequency and damping rate, respectively [15]. As the diffusion rate $D$ is the same in Eqs. $(9 a, b)$ and (10), the relative strengths of the polarization fluctuations as compared to the intensity fluctuations are approximately equal to the ratio of the relaxation decay rate $\gamma_{\text {ro }}$, over the polarization decay rate $\gamma_{0}$, where low damping corresponds to a sharp resonance and large fluctuations.

Once more it is relatively easy to generalize the expressions for the projected polarization noise to beyond the approximation of stable intensity. In the common case of relatively small intensity and polarization fluctuations ( $D$ $\left.\ll \gamma_{0}, \gamma_{\text {ro }}\right)$, the time-dependent part of the projected intensity is approximately $\frac{1}{2} \Delta I(t)+I_{0} \chi(t)$ or $\frac{1}{2} \Delta I(t)+I_{0} \phi(t)$, where $\Delta I(t)$ is the deviation from the average intensity $I_{0}$. As the intensity and polarization fluctuations are practically uncorrelated, apart from minor interactions via the dichroism $\gamma_{\text {lin }}$ and $\gamma_{\text {non }}$, the general projected noise spectrum is equal to the sum of the ideal polarization noise spectrum [Eqs. (9)] and the (scaled) intensity noise spectrum, as measured without polarization projection [Eq. (10)].

The difference between the $\phi$ and $\chi$ projections, i.e., between Eqs. (9a) and (9b), is a measure for the ellipticity of the noise cloud on the Poincaré sphere:

$$
\frac{\left\langle|\phi(\omega)|^{2}\right\rangle}{\left\langle|\chi(\omega)|^{2}\right\rangle}=\frac{\omega^{2}+\left(\omega_{\text {lin }}+2 \alpha \gamma_{\text {non }}\right)^{2}+\left(\gamma_{\|}+2 \gamma_{\text {non }}\right)^{2}}{\omega^{2}+\omega_{\text {lin }}^{2}+\gamma_{\|}^{2}},
$$

and can be used to estimate the nonlinear anisotropies $\gamma_{\text {non }}$ and $\alpha \gamma_{\text {non }}$. For relatively large linear birefringence $\left(\omega_{\text {lin }}\right.$ $\left.\gg \gamma_{\|}, \gamma_{\text {non }}, \alpha \gamma_{\text {non }}\right)$ the ratio displayed in Eq. (11) approaches unity and the exact result can be approximated as

$$
\frac{\left\langle|\phi(\omega)|^{2}\right\rangle^{1 / 2}}{\left\langle|\chi(\omega)|^{2}\right\rangle^{1 / 2}} \approx 1+\frac{2 \alpha \gamma_{\text {non }}}{\omega_{0}}\left(\frac{\omega_{0}^{2}}{\omega_{0}^{2}+\omega^{2}}\right)
$$

where we have introduced square roots to facilitate a comparison with the experimental signal on the RF analyzer [2]. Equation (12) shows that the nonuniformity of the polarization fluctuations depends on frequency, being relatively large for $\omega \leqslant \omega_{0}$ and disappearing for $\omega \gg \omega_{0}$. This aspect was apparently overlooked in the time domain analysis in [11], because that analysis neglected the nonorthogonality of the eigenvectors [Eq. (4)].

When linear birefringence is not the dominant anisotropy the analysis becomes more complicated. In principle one should use the exact result Eq. (11) instead of the approximate expression Eq. (12). A problem is that the exact result Eq. (11), which can be written as $\left(\omega^{2}+C_{\phi}\right) /\left(\omega^{2}+C_{\chi}\right)$, is complicated, because the $C$ coefficients contain many unknowns. A rewrite as

$$
\alpha \gamma_{\mathrm{non}}=\frac{\omega_{0}}{2} \frac{C_{\phi}-C_{\chi}}{C_{\phi}+C_{\chi}}\left(\frac{1+\left[2\left(\alpha^{2}+1\right) \gamma_{\mathrm{non}}^{2}+\gamma_{0}^{2}\right] / \omega_{0}^{2}}{\sqrt{1+\left(\alpha^{2}+1\right) \gamma_{\mathrm{non}}^{2} / \omega_{0}^{2}}+\gamma_{0} /\left(\alpha \omega_{0}\right)}\right)
$$

provides some help, as in practical cases (see Sec. VIII) the complicated factor within parentheses is generally very close to unity. In the experimental analysis we will first neglect this correction factor, and substitute the fitted $C_{\phi}$ and $C_{\chi}$ into Eq. (13) to derive the nonlinear anisotropy $\alpha \gamma_{\text {non }}$. As a next step we resubstitute the obtained result (and assume that $\alpha$ $\gg 1$ ) for a somewhat better second estimate.

Hofmann and Hess [11] already noted that the fluctuations in $\phi$ and $\chi$ are not independent, but correlated. As a result, the projected polarization noise will have extrema for directions different from the $\phi$ and $\chi$ axes. To find the rotation angle $\Psi_{\text {rot }}$, of the elliptical noise cloud in the $\phi, \chi$ plane, we rewrite Eqs. $(6 a, b)$ to obtain

$$
\begin{gathered}
\left\langle|\phi(\omega) \cos \Psi+\chi(\omega) \sin \Psi|^{2}\right\rangle \\
\propto\left[\omega^{2}+C_{0}+C_{1} \cos 2\left(\Psi-\Psi_{\text {rot }}\right)\right], \\
\tan \left(2 \Psi_{\text {rot }}\right)=\frac{\omega_{\text {lin }}-\alpha \gamma_{\|}}{\alpha \omega_{\text {lin }}+\gamma_{\|}+\left(\alpha^{2}+1\right) \gamma_{\text {non }}} \approx \frac{1}{\alpha},
\end{gathered}
$$

where $C_{0}$ and $C_{1}$ are constants, and where the approximation in Eq. (14b) is valid only in the limit of dominant linear birefringence $\left(\omega_{\text {lin }} \gg \alpha \gamma_{\|}, \gamma_{\text {non }}, \alpha \gamma_{\text {non }}\right)$. Note that the predicted rotation angle $\Psi_{\text {rot }}$ is independent of frequency; a change of detection frequency will only affect the ellipticity of the polarization noise cloud on the Poincare sphere, but not the angle $\Psi_{\text {rot }}$ at which the noise reaches its maximum.

\section{POLARIZATION FLUCTUATIONS FOR LARGE LINEAR BIREFRINGENCE}

The above analysis was based on a linearized description of the spin-eliminated model; i.e., the relative strength of the various anisotropies could be anything, as long as the laser polarization remained approximately linear $(\phi, \chi \ll 1)$. In practical VCSELs, the linear birefringence generally dominates over all other anisotropies, i.e., $\omega_{\text {lin }}$ $\gg \gamma_{\text {lin }}, \gamma_{\text {non }}, \alpha \gamma_{\text {non }}$, being still small enough to satisfy the adiabatic approximation, for which $\omega_{\text {lin }} \ll \gamma_{s} / \alpha$ is needed [6] [typical numbers are $\gamma_{\text {lin }}<3 \mathrm{~ns}^{-1}, \gamma_{\text {non }} \approx 1 \mathrm{~ns}^{-1}, \alpha \approx 3, \omega_{\text {lin }}$ $\approx 60 \mathrm{~ns}^{-1}$, and $\gamma_{s} \approx 300 \mathrm{~ns}^{-1}$ (see below and [6,9,23])]. For this common case of dominant linear birefringence the spineliminated model can be further simplified by a second adiabatic elimination, as demonstrated in this section.

We start by noting that, for the case of dominant linear 
birefringence, the polarization-resolved optical and intensity noise spectra become relatively simple, as the strength of the FWM peak and the nonuniformity of the polarization fluctuations are strongly reduced, being inversely proportional to $\omega_{0}^{2}$ and $\omega_{0}$, respectively [see Eqs. (8) and (12)]. One explanation for this behavior is that the relatively fast rotation on the Poincare sphere, associated with the large linear birefringence, makes all trajectories look like "tightly wound corkscrews" and thereby smooths out the difference between $\phi$ and $\chi$ dynamics. An equivalent explanation is that the large frequency difference, in the optical spectrum, between the nonlasing and lasing peak reduces the coupling between the two, making the orthogonal polarization mode look more and more like a standard nonlasing mode.

As a starting point for our full (nonlinearized) description of the polarization dynamics we could use Eqs. (A1) in Appendix A. Instead, it is more convenient to rewrite the spineliminated model in terms of the normalized Stokes vector, as [11]

$$
\begin{gathered}
\frac{d P_{1}}{d t}=\gamma_{\operatorname{lin}} \cos 2 \beta\left(1-P_{1}^{2}\right)-\gamma_{\operatorname{lin}} \sin 2 \beta P_{1} P_{2} \\
+2 \gamma_{\mathrm{non}} P_{1} P_{3}^{2}+2 \alpha \gamma_{\mathrm{non}} P_{2} P_{3} \\
\frac{d P_{2}}{d t}=-\omega_{\operatorname{lin}} P_{3}+\gamma_{\operatorname{lin}} \sin 2 \beta\left(1-P_{2}^{2}\right)-\gamma_{\operatorname{lin}} \cos 2 \beta P_{1} P_{2} \\
+2 \gamma_{\mathrm{non}} P_{2} P_{3}^{2}-2 \alpha \gamma_{\mathrm{non}} P_{1} P_{3} \\
\frac{d P_{3}}{d t}=\omega_{\operatorname{lin}} P_{2}-\gamma_{\operatorname{lin}} \cos 2 \beta P_{1} P_{3} \\
\quad-\gamma_{\operatorname{lin}} \sin 2 \beta P_{2} P_{3}-2 \gamma_{\mathrm{non}} P_{3}\left(1-P_{3}^{2}\right)
\end{gathered}
$$

For the case of dominant linear birefringence the prevailing evolution over the Poincare sphere is a fast rotation around the $P_{1}$ axis, where $P_{2}$ and $P_{3}$ perform a rapid out-of-phase oscillation with approximate frequency $\omega_{\operatorname{lin}}$, driven by the first terms in Eqs. $(15 \mathrm{~b}, \mathrm{c})$. On top of this rapid oscillation of the $P_{2}$ and $P_{3}$ coordinates, there is a much slower evolution of the $P_{1}$ coordinate, that can be separated out via a new adiabatic elimination. On the Poincare sphere, the slow variable measures the position of an almost circular orbit at almost constant $P_{1}=\cos (2 \varphi)$, where $\varphi=\phi$ only at $\chi=0$. By averaging Eq. (15a) over the fast rotation just mentioned, we can set $\left\langle P_{1} P_{2}\right\rangle \approx 0, \quad\left\langle P_{2} P_{3}\right\rangle \approx 0, \quad$ and $\left\langle P_{1} P_{3}^{2}\right\rangle$ $\approx(1 / 2) P_{1}\left(1-P_{1}^{2}\right)$, to obtain

$$
\frac{d P_{1}}{d t} \approx\left(\gamma_{\|}+\gamma_{\text {non }} P_{1}\right)\left(1-P_{1}^{2}\right) .
$$

As the combination $\left(1-P_{1}\right) / 2$ is equal to the relative intensity of the $y$-polarized light, the above equation describes the deterministic evolution that underlies the polarization-mode partition noise.

To obtain the full polarization dynamics we will now add noise to the above equation (16). For the angle $\varphi$ it is immediately clear how much noise should be added: as polarization noise is isotropic on the Poincare sphere, the amount of noise $f_{\varphi}$, perpendicular to the fast orbital evolution, is equal to that in the other projections $f_{\chi}$ and $f_{\phi}$ [see Eq. (5)]. The amount of noise in $P_{1}$ is then found by a simple transformation. The addition of noise can also produce extra drift terms in the equations [18]. For instance, the polarization noise in $P_{2}$ and $P_{3}$ will produce a steady decrease of $P_{1}^{2}=1-P_{2}^{2}$ $-P_{3}^{2}$. Keeping this into account we obtain the following stochastic equations:

$$
\begin{gathered}
\frac{d P_{1}}{d t}=\left(\gamma_{\|}+\gamma_{\text {non }} P_{1}\right)\left(1-P_{1}^{2}\right)-4 D P_{1}+\left(2 \sqrt{1-P_{1}^{2}}\right) f_{\varphi}, \\
\frac{d \varphi}{d t}=-\frac{\gamma_{\|}}{2} \sin (2 \varphi)-\frac{\gamma_{\text {non }}}{4} \sin (4 \varphi)+\frac{D}{\tan (2 \varphi)}+f_{\varphi} .
\end{gathered}
$$

These equations show how the dominant linear birefringence, or fast rotation on the Poincare sphere, effectively redirects the nonlinear anisotropy, so that the original (nonlinear) competition between the two circularly polarized states is converted into a competition between the linearly polarized states aligned along the axes of birefringence. Equation (17b) thus has the same form as Eq. (9) in [19], which was recently derived for the dynamics of the ellipticity angle $\chi$ of an isotropic class A laser with strong competition between its circularly polarized fields.

By transforming the above equations $(17 \mathrm{a}, \mathrm{b})$ into the corresponding Fokker-Planck equations we regain the standard problem of "diffusion in a potential well," on which the dynamics of a class A laser is usually mapped [20,21]. The steady-state probability distributions and potentials of our system are

$$
\begin{gathered}
P\left(P_{1}\right) \propto \exp \left[-\frac{V_{P_{1}}\left(P_{1}\right)}{D}\right] \propto \exp \left[\frac{\gamma_{\|}}{2 D} P_{1}-\frac{\gamma_{\text {non }}}{4 D}\left(1-P_{1}^{2}\right)\right], \\
P(\varphi) \propto \exp \left[-\frac{V_{\varphi}(\varphi)}{D}\right] \\
\propto ⿻ \sin (2 \varphi) \exp \left[\frac{\gamma_{\|}}{2 D} \cos (2 \varphi)+\frac{\gamma_{\text {non }}}{8 D} \cos (4 \varphi)\right] .
\end{gathered}
$$

The above result can be used to calculate the power ratio of the nonlasing and lasing mode $P_{\text {nonlasing }} / P_{\text {lasing }}$, or, equivalently, the mean-square deviation from the steadystate polarization, or, equivalently, the size of the noise cloud on the Poincaré sphere [see Eq. (1)]. For dominant $x$-polarized emission one finds

$$
\frac{P_{\text {nonlasing }}}{P_{\text {lasing }}}=\frac{1}{2}\left(1-\left\langle P_{1}\right\rangle\right)=\left\langle\varphi^{2}\right\rangle=\frac{D}{\gamma_{\|}+\gamma_{\text {non }}}=\frac{D}{\gamma_{0}} .
$$

For dominant $y$ polarization the expression is the same, apart from a minus sign in front of $\gamma_{\|}$. Note that integration of the projected polarization noise spectrum, Eqs. (9), over (positive and negative) frequency, gives the same result, for the case of dominant linear birefringence considered here. Equation (19) shows, in a very convenient way, how polarization fluctuations result from a balance between a stochastic force 
on the one hand and the restoring forces of the (absorptive) anisotropies on the other hand. More specifically, it shows how the relative power in the nonlasing polarization, or the size of the noise cloud on the Poincare sphere, can be used to estimate the noise strength $D$, when the dichroism $\gamma_{0}$ is known.

Polarization noise can make the laser hop from the potential well of dominant $x$ polarization to the other well of dominant $y$ polarization, and back. The present model gives a simple expression for the average hopping time if $\gamma_{\text {non }} /(4 D) \gg 1$. For the symmetric case $\left(\gamma_{\|}=0\right)$ the average dwell time in each state is given by $[20,21]$

$$
\langle T\rangle \approx \sqrt{\frac{\pi D}{\gamma_{\mathrm{non}}}} \frac{1}{\gamma_{\mathrm{non}}} e^{\left[\gamma_{\mathrm{non}} /(4 D)\right]} .
$$

In the limit $\gamma_{\text {non }} /(4 D) \gg 1$, the above expression for the hopping time is extremely sensitive to the polarization diffusion rate $D$, so that it can be used to get an accurate measure thereof, once $\gamma_{\text {non }}$ is known.

For completeness we note that, in the spin-eliminated model, there are actually two different mechanisms that can produce a polarization switch. One type of switch occurs when we let the linear dichroism $\gamma_{\|}$depend on injection current [7,9], in such a way that $\gamma_{\|}$changes sign at a certain current; at this point $\gamma_{\|} \approx 0$ and lasing in the two polarization directions is equally favorable. This first type of polarization switch should obey the equations in this section, at least for the (common) case of dominant linear birefringence. Depending on the amount of noise $D$ and the strength of the nonlinear dichroism $\gamma_{\text {non }}$, the laser polarization will exhibit fast, or slow hopping [see Eq. (20)], where extremely slow hopping will experimentally be interpreted as bistability or hysteresis. The second type of switch is not based on currentdependent linear effects, but has an intrinsic nonlinear nature. In the spin-eliminated model, Eq. (3b) shows how this nonlinear switch can occur only in VCSELs with small negative linear birefringence $\omega_{\text {lin }}$, where the nonlinear redshift can pull the (high-frequency) nonlasing mode into the lasing mode, to create polarization instability and switching [6]. For $\omega_{0}^{2}<-\gamma_{0}^{2}$ one of the eigenvalues $-\gamma_{0} \pm i \omega_{0}$ will correspond to an undamped evolution, the polarization fluctuations will become excessively large in one direction, and one has to go beyond the linearized equations to solve the problem. This phenomenon has been discussed in many theoretical papers, e.g., in terms of a Hopf bifurcation towards elliptically polarized modes [4,5], although the exact nature of this switch is often hidden in complicated carrier dynamics. In the experiments, linear birefringence generally dominates over the other anisotropies so that this second type of polarization switch, with its different behavior and different statistics, is quite rare [22].

\section{EXPERIMENTAL SETUP}

For the experiments we have used a batch of some 50 proton-implanted VCSELs, organized as 1D arrays. The lasers operate around $850 \mathrm{~nm}$ and comprise three 8-nm-thick GaAs quantum wells in a $1 \lambda$ cavity, sandwiched between an upper and lower Bragg mirror of 19 and 29.5 layer pairs, respectively [24]. The threshold currents of all these

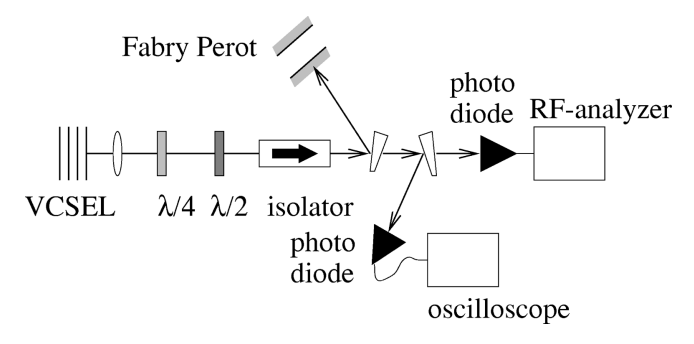

FIG. 2. Experimental setup. After polarization projection we measure (i) optical spectra with a Fabry-Pérot interferometer, (ii) projected noise spectra with a 6-GHz photodiode and $\mathrm{RF}$ analyzer, and (iii) time traces with a fast photodiode and oscilloscope.

VCSELs is around $5 \mathrm{~mA}$, with higher-order modes appearing around $10 \mathrm{~mA}$ at an output power of about $2 \mathrm{~mW}$. At low current the laser polarization was practically always close to vertical, i.e., perpendicular to the array axis. The steady-state ellipticity $\chi_{\mathrm{ss}}$ was typically $1^{\circ}$ or less, with a few exceptions of $\chi_{\mathrm{ss}} \approx 5-10^{\circ}$ for lasers with small negative birefringence $\omega_{0}$. The size of the batch allowed us to pick the most interesting VCSELs for further study, namely, those with relatively small effective birefringence and those that exhibit a polarization switch. In the presentation of the figures we will concentrate on two specific VCSELs, which we have labeled VCSEL 1 and VCSEL 2. Unfortunately, the (currentdependent) VCSEL performance showed small variations from day to day, so that the exact numbers for birefringence and dichroism, as obtained for the same VCSEL from the various figures, do not always match.

The experimental setup is sketched in Fig. 2. To limit the external noise to the minimum, the VCSEL is enclosed in a temperature-stabilized box (stability $\approx 0.1 \mathrm{mK}$ ) and driven by a stable current source (stability $\approx 0.75 \mu \mathrm{A}$ from dc to 1 $\mathrm{MHz})$. The collimated laser light is first passed through a (rotatable) $\lambda / 4$ plate, and subsequently through a combination of a (rotatable) $\lambda / 2$ plate and optical isolator, which together effectively act as a rotatable polarizer. By setting the angles of the $\lambda / 4$ and $\lambda / 2$ plates we select the polarization state on which the laser light is projected. After projection the light can be analyzed in three different ways. A planar Fabry-Pérot interferometer, with adjustable free spectral range, allows for detailed measurements of the optical spectrum. A 6-GHz low-noise photoreceiver (NewFocus 1534), in combination with a $25-\mathrm{GHz}$ RF analyzer (HewlettPackard HP0563E), allows for measurements of the (polarization-resolved) intensity noise. As a third method we can also observe this noise in the time domain, using a fast photodiode (DC-200 MHz) in combination with a 350-MHz oscilloscope (LeCroy 9450). In the next sections we will discuss the results of these three methods in consecutive order.

\section{POLARIZATION-RESOLVED OPTICAL SPECTRA}

Figure 3 shows optical spectra, for VCSEL 1 operating at $I=9.0 \mathrm{~mA}$. In Fig. 3(a) the wave plates were set for projection onto the dominant (horizontal) polarization, whereas this polarization was largely blocked in Fig. 3(b) (we intentionally kept a very small fraction of the lasing peak to serve as a marker). These figures show that the optical spectrum consists of three (equidistant) peaks, which (from left to right) 


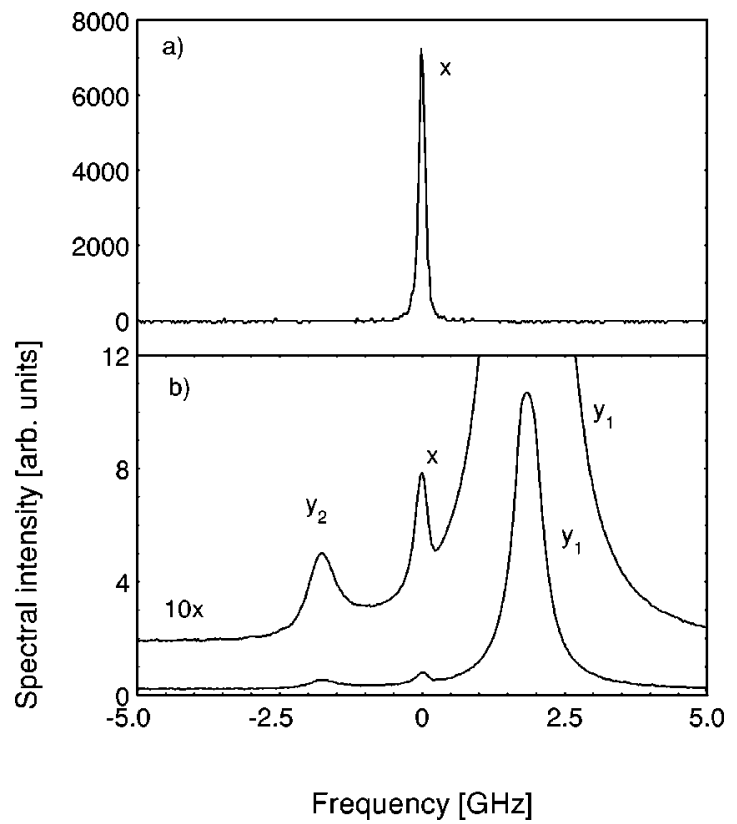

FIG. 3. Polarization-resolved optical spectra of VCSEL 1 at $I$ $=9.0 \mathrm{~mA}$, taking with a Fabry-Pérot interferometer. The $x$-polarized lasing peak, which dominates (a), is almost completely suppressed in the $y$-polarized spectrum of (b) (same arbitrary units). The latter shows the nonlasing peak at higher frequency and a weak FWM peak, as mirror image, at lower frequency.

are denoted the four-wave-mixing (FWM) peak $\left(y_{2}\right)$, the lasing peak $(x)$, and the nonlasing peak $\left(y_{1}\right)$. Roughly speaking, the lasing peak is associated with the steady-state polarization of the laser, the nonlasing peak is a result of amplified spontaneous emission in the orthogonal polarization, and the four-wave-mixing peak results from nonlinear mixing between these two. Comparison of the vertical scale of Figs. 3(a) and 3(b) shows that the lasing peak dominates over the nonlasing peak by roughly 3 orders of magnitude; it takes quite some suppression to resolve the latter. The FWM peak is much weaker still and often difficult to observe. In fact its presence was first reported only recently [2].

The optical spectra of Figs. 3(a) and 3(b) contain information about many laser parameters. First of all the frequency difference between the lasing and nonlasing peak gives the effective birefringence $\omega_{0}$, whereas the difference in their HWHM spectral width gives the effective dichroism $\gamma_{0}$. For VCSEL 1 studied in Fig. 3, the effective birefringence is relatively small at $\nu_{0} \equiv \omega_{0} /(2 \pi)=-1.82(2) \mathrm{GHz}$ (minus sign because the low-frequency mode lases); this is why it has been selected. Its effective dichroism has a more typical value, namely $\gamma_{0} /(2 \pi)=0.22(2) \mathrm{GHz}$. For most other VCSELs $\nu_{0}$ ranged between -3 and $+15 \mathrm{GHz}$ (with two exceptions at +25 and $+40 \mathrm{GHz}$ ); the dichroism $\gamma_{0} /(2 \pi)$ was always below $1 \mathrm{GHz}$. In Fig. 3 the measured spectral width of the lasing mode is instrument limited to $0.06 \mathrm{GHz}$ (HWHM) by the resolution of the Fabry-Pérot interferometer.

Equation (8) shows how the relative strength of the fourwave-mixing (FWM) peak, as compared to the nonlasing peak, can be used to quantify the nonlinear anisotropies in the laser. From Fig. 3(b) we find this relative strength to be $2.5(2) \%$. With $\nu_{0}=-1.82(2) \mathrm{GHz}$ this gives a combined

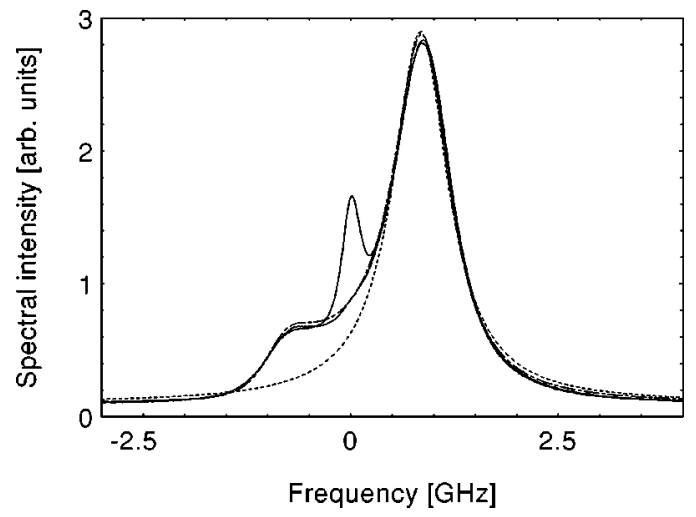

FIG. 4. The optical spectrum of VCSEL 2 at $I=10.0 \mathrm{~mA}$ shows how, for VCSELs with very small birefringence $\left(\nu_{0}=-0.85 \mathrm{GHz}\right.$ in the present case) the FWM peak can be as much as $20 \%$ of the nonlasing peak. The dashed-dotted and dotted lines are fits to Eq. (7) and to a single Lorentzian, respectively.

nonlinear anisotropy of $\sqrt{\alpha^{2}+1} \gamma_{\mathrm{non}}=3.6(2) \mathrm{ns}^{-1}$. Unfortunately, the optical spectrum does not allow a further separation into nonlinear birefringence and nonlinear dichroism; it mainly provides information on the nonlinear birefringence, as generally $\alpha \gg 1$ [25], so that $\sqrt{\alpha^{2}+1} \approx \alpha$.

Theoretically we expect the relative strength of the FWM peak (as compared to the nonlasing peak) to be inversely proportional to the square of the effective birefringence $\omega_{0}$ [see Eq. (8)]. This is indeed observed: for two other VCSELs we measured a relative strength of $0.63(7) \%$ at $\nu_{0}$ $=3.45 \mathrm{GHz}$, and $0.15(3) \%$ at $\nu_{0}=6.7 \mathrm{GHz}$. For our "average" VCSEL, with $\nu_{0} \approx 10 \mathrm{GHz}$, the strength of the FWM peak was below $0.1 \%$ of that of the nonlasing peak and thereby below the noise level. This strong dependence on birefringence explains why the FWM peak was not noticed until recently.

As a last piece of information we calculate the amount of polarization fluctuations, by dividing the sum of the spectrally integrated strengths of $y$-polarized nonlasing and FWM peak by the (integrated) $x$-polarized lasing peak. From Fig. 3 we determine this ratio to be $0.65(5) \%$. On the Poincare sphere, this corresponds to a noise cloud with a size $\left\langle(2 \varphi)^{2}\right\rangle^{1 / 2} \approx 9^{\circ}$ [see Eq. (19)], which, on the world globe, is equivalent to an area bigger than Alaska, but smaller than Australia. At the end of Sec. VIII we will discuss how the above value can be used to determine the magnitude of the polarization noise, and thereby the cavity loss rate $\kappa$.

For VCSEL 2, studied in Fig. 4, the birefringence is extremely small (and negative) at $\nu_{0}=-0.87 \mathrm{GHz}$ in VCSEL. As a consequence, the strength of the FWM peak now amounts to about $20 \%$ of that of the nonlasing peak. For this extreme situation the nonlinear and linear anisotropies are comparable in strength and the nonlinear effect can no longer be treated as a weak perturbation. However, even for this extreme situation, the linearized theory developed in Sec. III remains valid; the relative strength of the nonlasing and FWM peak, as compared to the lasing peak, is still only $\approx 1 \%$, so that $\phi, \chi \ll 1$. This is demonstrated by the dasheddotted curve in Fig. 4, which is a fit of Eq. (7) to the optical spectrum, where the fitted width includes the finite width of the lasing peak. The dotted curve shows the Lorentzian fit to the nonlasing peak only. 


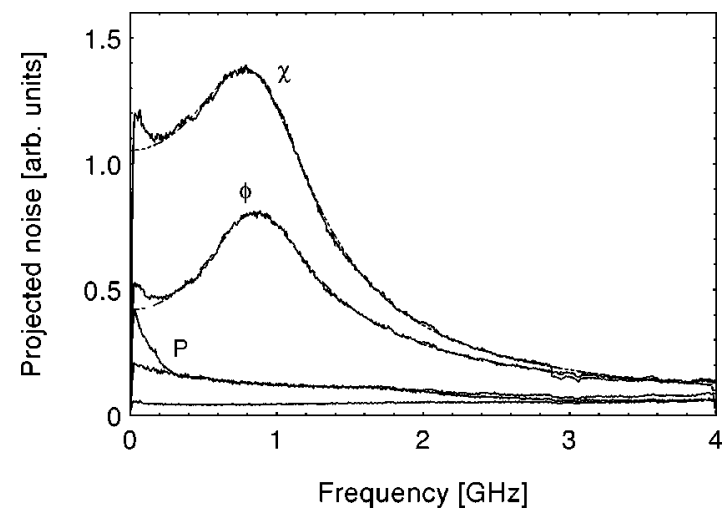

FIG. 5. Projected intensity noise of VCSEL 2 at $I=9.0 \mathrm{~mA}$. From top to bottom the curves show noise spectra for projection onto the $\chi$ direction, onto the $\phi$ direction, onto the lasing polarization (label $P$ ), onto the nonlasing polarization, and the noise in the absence of light (system limit).

\section{POLARIZATION-RESOLVED INTENSITY NOISE SPECTRA}

In this section we will describe measurements of the polarization-resolved intensity noise, for which the principle was already discussed in Sec. IV (see Fig. 1). The practical implementation is based on a spectral analysis of the intensity noise of laser light that has passed through a rotatable $\lambda / 4$ plate and a combination of a rotatable $\lambda / 2$ and isolator, which together act as a rotatable polarizer (see Fig. 2). Figure 5 shows spectra of the projected intensity noise $\left\langle\left|I_{\text {project }}(\omega)\right|^{2}\right\rangle^{1 / 2}$ for VCSEL 2 operating at $I=9.0 \mathrm{~mA}$, with a relatively small birefringence of $\nu_{0}=-0.85 \mathrm{GHz}$. From top to bottom, the curves in Fig. 5 show noise spectra for projection onto the $\chi$ direction, onto the $\phi$ direction, onto the lasing polarization (label $P$ ), onto the nonlasing polarization, and the noise in the absence of light (system limit). As the noise in the first two projections is much larger than that for projection onto the lasing polarization, our first conclusion is that polarization noise dominates over pure intensity noise. Our analysis will concentrate on the noise spectra observed for the $\chi$ and $\phi$ projections.

The dashed curves in Fig. 5 are fits of Eq. (9) to the upper two experimental curves over the range $0.3-2.5 \mathrm{GHz}$. The fitting range has been limited to avoid both the lowfrequency noise tail, as well as the high-frequency noise floor. The high quality of the fits allows us to extract the effective birefringence $\omega_{0}$, the effective dichroism $\gamma_{0}$, a constant $C$ [used to simplify the numerator of Eq. (9) to $\omega^{2}+C$, see also the discussion just above Eq. (13)], and a proportionality constant, which contains the detected intensity $I$, the diffusion rate $D$, and the system response. Our fitting results are $\left|\nu_{0}\right|=\left|\omega_{0} /(2 \pi)\right|=0.85(2) \mathrm{GHz}$, $\gamma_{0} /(2 \pi)=0.38(2) \mathrm{GHz}, \quad C_{\phi} /\left(4 \pi^{2}\right)=0.49 \mathrm{GHz}^{2}, \quad$ and $C_{\chi} /\left(4 \pi^{2}\right)=3.6 \mathrm{GHz}^{2}$. The first two parameters, $\nu_{0}$ and $\gamma_{0}$, can also be obtained from optical spectra. A big advantage of the present measurement is its extreme resolution: a spectral analysis of intensity noise is only limited by the resolution of the RF analyzer, which can easily be below $1 \mathrm{kHz}$, whereas optical measurements are limited by the Fabry-Pérot resolution of typically $10-100 \mathrm{MHz}$.

Figure 5 shows that the projected intensity noise in the $\chi$

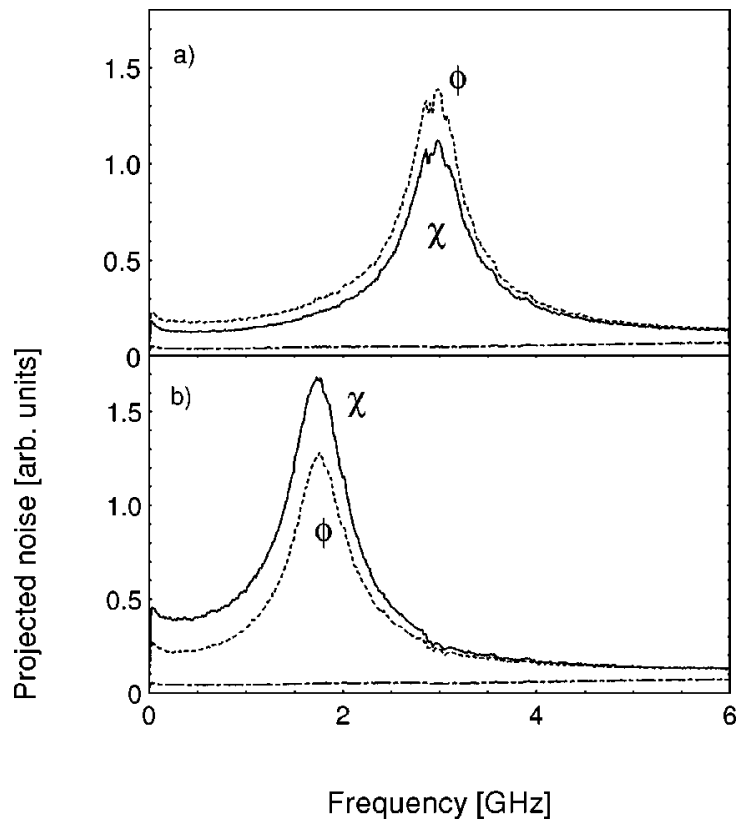

FIG. 6. Projected intensity noise for VCSEL 1 before and after a polarization switch, at (a) $I=8.5 \mathrm{~mA}$, and (b) $I=9.0 \mathrm{~mA}$.

direction is much bigger than that in the $\phi$ direction $\left(C_{\chi}\right.$ $>C_{\phi}$ ), or, in other words, that the polarization fluctuations are highly nonuniform and that the noise cloud on the Poincaré sphere is elliptical instead of circular. This difference is intimately related to the presence of the FWM peak in the optical spectrum, and can likewise be used to estimate the strength of the nonlinear anisotropies. To do so we determine the ratio $\left\langle|\phi(\omega)|^{2}\right\rangle^{1 / 2} /\left\langle|\chi(\omega)|^{2}\right\rangle^{1 / 2}$ and compare the result with Eqs. (11), (12), and (13). At the resonance frequency of $0.85 \mathrm{GHz}$ we find $\left\langle|\phi(\omega)|^{2}\right\rangle^{1 / 2} /\left\langle|\chi(\omega)|^{2}\right\rangle^{1 / 2}=0.59$. Substitution of this ratio in Eq. (12) yields $\alpha \gamma_{\text {non }} \approx 2.2 \mathrm{~ns}^{-1}$. As the very small birefringence makes the use of this approximate expression disputable, it is better to substitute the fitted $C_{\phi}$ and $C_{\chi}$ in Eq. (13), using the procedure discussed in Sec. IV. This yields estimates of $\alpha \gamma_{\text {non }} \approx 2.0 \mathrm{~ns}^{-1}$ on the first try and $\alpha \gamma_{\text {non }} \approx 2.5 \mathrm{~ns}^{-1}$ upon iteration.

The noise spectra observed for the projections onto the lasing and nonlasing polarization contain information on the intensity and polarization partition noise. A detailed analysis of these spectra will be published elsewhere [26]. The relative strength of the various noise spectra shows how the $\chi$ and $\phi$ projection are first order in the polarization fluctuations and how the projections onto the lasing and nonlasing polarization are only second order.

Figure 6 shows spectra of the projected intensity noise of VCSEL 1. This VCSEL exhibits a polarization switch; it operates on the high-frequency (vertically polarized) mode at $I=8.5 \mathrm{~mA}$ [Fig. 6(a)] and on the low-frequency (horizontally polarized) mode at $I=9.0 \mathrm{~mA}$ [Fig. 6(b)]. In both figures the solid and dashed curves denote the intensity noise for projection onto the $\chi$ and $\phi$-direction, respectively, whereas the dash-dotted curve shows the system noise floor. The fits to these noise spectra (not shown) were again excellent and gave $\left|\nu_{0}\right|=2.96(2) \mathrm{GHz}, \quad \gamma_{0} /(2 \pi)=0.23(2)$ $\mathrm{GHz}$, and $\alpha \gamma_{\mathrm{non}}=2.8(3) \mathrm{ns}^{-1}$ at $I=8.5 \mathrm{~mA}$, and $\left|\nu_{0}\right|$ $=1.75(2) \mathrm{GHz}, \quad \gamma_{0} /(2 \pi)=0.23(2) \mathrm{GHz}, \quad$ and $\quad \alpha \gamma_{\text {non }}$ $=3.2(3) \mathrm{ns}^{-1}$ at $I=9.0 \mathrm{~mA}$. In Fig. 6 the differences be- 


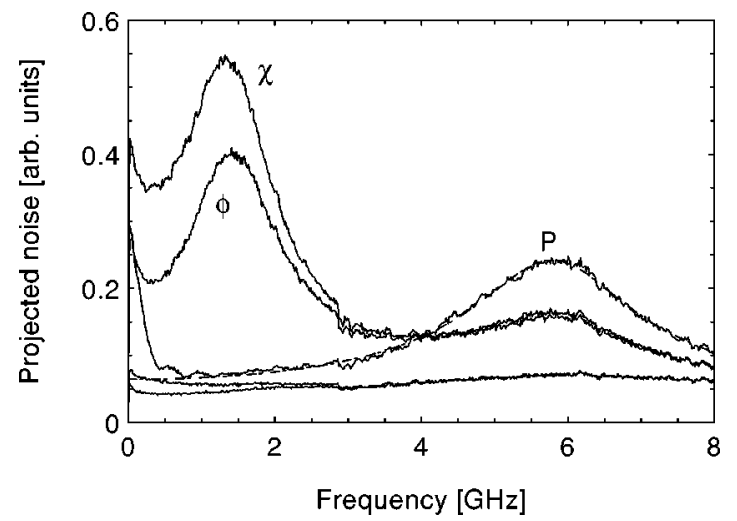

FIG. 7. Projected intensity noise for VCSEL 2 at $I=7.0 \mathrm{~mA}$. Note the presence of the relaxation oscillations around $6 \mathrm{GHz}$ in the projection onto the lasing polarization (label $P$ ) and the corresponding structure in the polarization-resolved intensity noise $(\phi$ and $\chi)$. The dashed curve is a fit based on Eq. (10).

tween $\phi$ and $\chi$ noise are less prominent than in Fig. 5 as a result of the larger birefringence. The main message of this figure is that the nonuniformity of the polarization fluctuation is as expected for $\alpha \gg 1$; when the high-frequency mode lases we find $|\phi(\omega)|>|\chi(\omega)|$ [Fig. 6(a)]; when the lowfrequency mode lases we find $|\phi(\omega)|<|\chi(\omega)|$ [Figs. 5 and $6(\mathrm{~b})]$.

Figure 7 shows again the projected intensity noise of VCSEL 2 (as in Fig. 5), but now at an operating current of $I=7.0 \mathrm{~mA}$, i.e., closer to threshold $\left(I_{\mathrm{th}}=5.0 \mathrm{~mA}\right)$, and for a wider frequency range. The spectrum for projection onto the lasing polarization (solid curve, label $P$ ) is dominated by pure intensity noise; the broad structure around $6 \mathrm{GHz}$ results from intensity fluctuations associated with the relaxation oscillations. The dashed-dotted line shows a fit of Eq. (10) to this noise spectrum, yielding a relaxation oscillation frequency of $5.8 \mathrm{GHz}$ and a damping (HWHM) of $1.1 \mathrm{GHz}$. The $\phi$ and $\chi$ curves show the noise spectra for projection onto the corresponding polarization states. From fits in the range $0.4-2.8 \mathrm{GHz}$ we find $\left|\nu_{0}\right|=1.39 \mathrm{GHz}, \gamma_{0} /(2 \pi)$ $=0.55 \mathrm{GHz}$, and $\alpha \gamma_{\text {non }}=2.2 \mathrm{~ns}^{-1}$. This figure clearly shows how intensity noise and polarization noise simply add up in the projection spectrum; the relaxation oscillation is of course less prominent in the $\phi$ and $\chi$ curves because the average intensity for polarization projection is about half the intensity for projection onto the lasing polarization.

Next we have measured the correlation between the polarization noise in $\phi$ and $\chi$, which, according to Sec. IV and [11], should be noticeable as a rotation of the elliptical noise cloud on the Poincaré sphere. For best results we took VCSEL 2, with its relatively small birefringence and large nonuniformity, and operated it at $9.0 \mathrm{~mA}$. Figure 8 shows a measurement series of the projected intensity noise as a function of the angle of the projecting polarizer, where $0^{\circ}$ and $45^{\circ}$ correspond to projection onto the $\chi$ and $\phi$ direction, respectively (see dashed vertical lines). The solid curve is a fit, using the square root of Eq. (14a). Figure 8 shows that the cases of maximum and minimum projection noise do not correspond to pure $\chi$ and $\phi$ projection, but occur at a slightly smaller angle. Specifically, the noise ellipse is rotated over an angle of $\Psi_{\text {rot }}=18(6)^{\circ}$ with respect to the $\chi, \phi$ coordinate

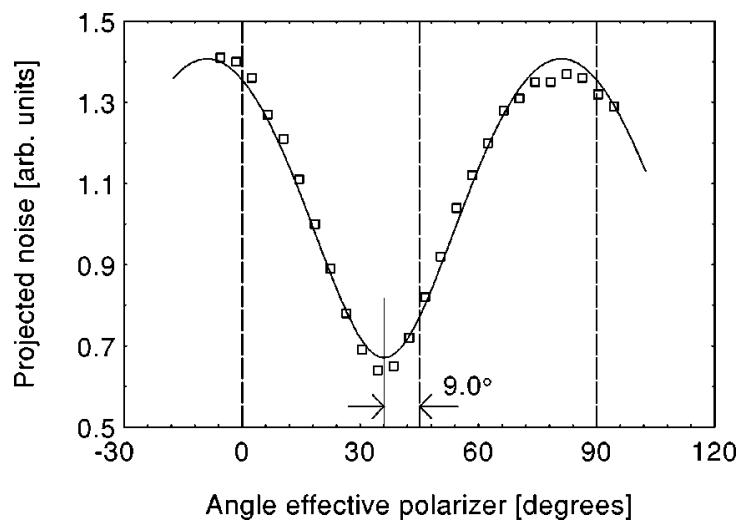

FIG. 8. Measurement of the projected intensity noise for VCSEL 2 at $I=9.0 \mathrm{~mA}$ as a function of the orientation angle of the projecting polarization. The angles $0^{\circ}$ and $45^{\circ}$ correspond to projection onto the $\chi$ and $\phi$ direction, respectively. Note the angular shift of about $-9^{\circ}$.

system. This agrees very roughly with the rotation angle as expected from Eq. (14b), which is about $9^{\circ}$ for the case of dominant birefringence $(\alpha=3)$, but as much as $36^{\circ}$ for the case at hand $\left[\omega_{\text {lin }} /(2 \pi)=-0.85 \mathrm{GHz}, \gamma_{\text {non }} \approx 1.0 \mathrm{~ns}^{-1}, \alpha\right.$ $\left.\approx 3, \gamma_{\|} \approx 1.4 \mathrm{~ns}^{-1}\right]$, where the latter estimate is clearly hindered by the uncertainties in the various parameters.

\section{POLARIZATION SWITCHES}

For some VCSELs the polarization direction changes suddenly by about $90^{\circ}$ when the laser current is varied. A study of the laser dynamics around such a polarization switch is ideally suited to determine the various laser parameters. This is demonstrated in Figs. 9(a) and 9(b), which show the effective birefringence $\left|\nu_{0}\right|$ and dichroism $\left|\gamma_{0}\right| /(2 \pi)$ of VCSEL 1, as obtained from the polarization-resolved intensity noise spectra, as a function of current. This VCSEL exhibits a polarization switch between 8.9 and $9.1 \mathrm{~mA}$. To be more specific: at low current the (vertically polarized) highfrequency mode lases, at high current the (horizontally polarized) low-frequency mode lases, whereas either situation can occur within the switching region, depending on history (hysteresis). Figure 9(a) shows how the frequency splitting between the lasing and nonlasing mode changes from $\left|\nu_{0}\right|$ $=3.16 \mathrm{GHz}$ to $1.93 \mathrm{GHz}$, when the VCSEL switches polarization. This change is a result of nonlinear birefringence and can be used as a measure thereof [2]. By expanding Eq. (3a) into a linearized expression for the "spectral redshift of the nonlasing mode" we deduce from the switch that $\alpha \gamma_{\text {non }} \approx \pi(3.16-1.93) \mathrm{ns}^{-1}=3.9 \mathrm{~ns}^{-1}$. Using the full Eq. (3a) we get a somewhat better estimate, $\alpha \gamma_{\text {non }} \approx 3.7 \mathrm{~ns}^{-1}$. We note that VCSEL 1 was also used to obtain the optical spectrum of Fig. 3 (at $I=9.0 \mathrm{~mA}$ and $\nu_{0}<0$, i.e., after the switch), and the polarization-resolved intensity noise of Fig. 6 (before and after the switch).

Figure 9(b) shows how the effective dichroism changes with current and how the vertically polarized mode becomes less and less dominant. This is a general trend in all our VCSELs: before the switch the dominant polarization is always close to vertical, i.e., perpendicular to the array axis; after the switch the dominant polarization becomes horizon- 


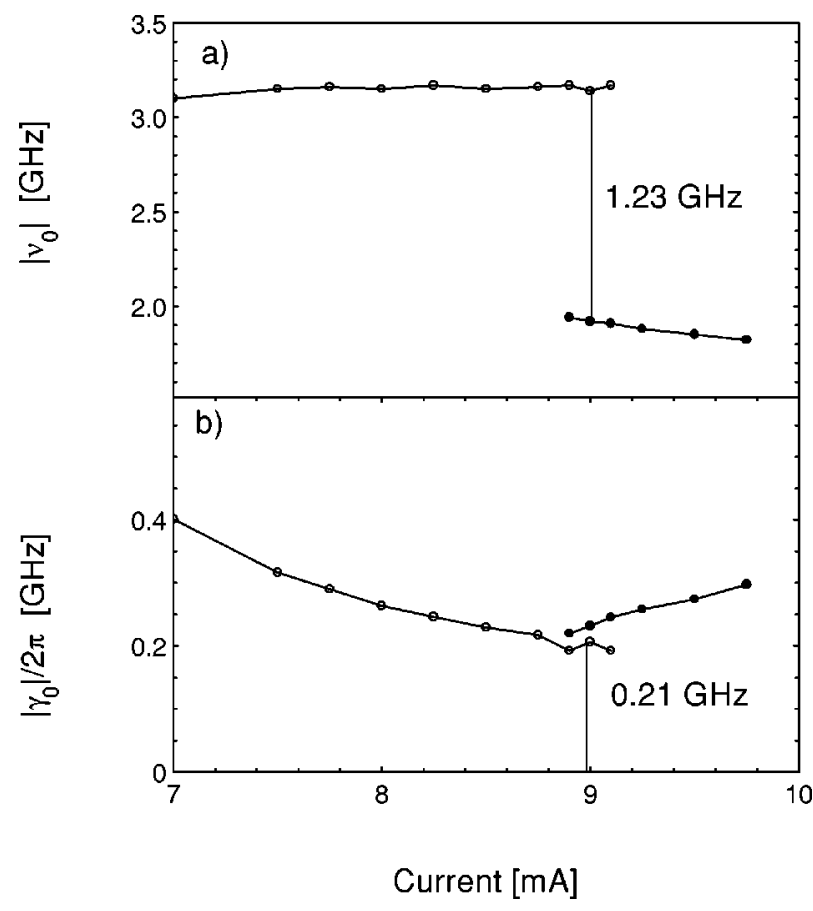

FIG. 9. The effective birefringence $\left|\nu_{0}\right|$ and dichroism $\left|\gamma_{0}\right|$ of VCSEL 1 as a function of current. Note the observed hysteresis and the jump in $\left|\nu_{0}\right|$ that occurs upon a polarization switch (around $I$ $=9.0 \mathrm{~mA}$ ). From (b) we conclude that the polarization switch results from a current dependence dichroism, $\gamma_{0}(I)$.

tal. Furthermore, VCSELs that have a small dichroism at low current exhibit a polarization switch at increasing current, whereas those with larger dichroism do not switch within the realm of fundamental mode operation. We therefore attribute the occurrence of these switches to a current dependence of the measured effective dichroism $\gamma_{0}(I)$, and more specifically to the linear part thereof, i.e., $\gamma_{\|}(I)$, as the nonlinear part $\gamma_{\text {non }}>0$ will always favor the lasing polarization over the nonlasing one and increase monotonically with current. A measurement of $\gamma_{0}(I)$ in fact allows us to predict whether or not a polarization switch is going to occur at a certain current. In the switching region the two polarizations will have almost equal loss $\left(\gamma_{\|} \approx 0\right)$ so that we conclude for the nonlinear dichroism $\gamma_{\text {non }} \approx \gamma_{0} \approx 2 \pi \times 0.21 \mathrm{~ns}^{-1}=1.3 \mathrm{~ns}^{-1}$ [see Fig. 9(b)]. Division of the nonlinear birefringence [in Fig. 9(a)] by the nonlinear dichroism [in Fig. 9(b)] yields $\alpha$ $\approx 2.9$, in agreement with literature values. Similar values were found for other VCSELs. As an example, one of these other VCSELs switched its polarization around $I=8.5 \mathrm{~mA}$, had a frequency splitting of $11.52 \mathrm{GHz}$ and $10.50 \mathrm{GHz}$ before and after the switch and an effective dichroism of $\gamma_{0} /(2 \pi)=0.22 \mathrm{GHz}$ within the switching region, so that $\alpha$ $\approx 3.1$.

We have thus demonstrated how a comparison of spectra before and after a polarization switch allows one to separately determine the nonlinear birefringence and nonlinear dichroism, irrespective of the VCSEL's absolute birefringence. In this respect the analysis presented here is more powerful then that in Secs. VII and VIII, addressing optical spectra and projected intensity noise; the latter approach worked only for small $\nu_{0}$ and gave only a value for the combined nonlinear birefringence and dichroism. A disadvantage, however, of the present technique is that the VCSEL should actually switch polarization and that one can determine the nonlinearities for only one specific current, being the switching current.

In practice, the VCSELs that switch their polarization can have both positive and negative effective birefringence $\nu_{0}$. In both cases, the observed changes in $\nu_{0}$ were consistent with the expected nonlinear redshift [see Eq. (3b)]: when the high-frequency mode dominates $\left(\nu_{0}>0\right)$ at low current, as is generally the case in our VCSELs, $\left|\nu_{0}\right|$ increased gradually with current and jumped to a smaller value upon a polarization switch; when the low-frequency mode is dominant $\left(\nu_{0}\right.$ $<0),\left|\nu_{0}\right|$ decreased with current, to jump to larger values upon a switch. Furthermore, switches have been observed in VCSELs with both small and large $\nu_{0}$. These observations show that the nonlinear anisotropies by themselves are not the prime reason for the occurrence of polarization switches, as the "nonlinear" explanation predicts only switches from low to higher frequency operation, and only at relatively small (negative) $\nu_{0}[4,8]$.

The physical mechanism behind the polarization switches, i.e., the mechanism responsible for the experimentally observed current dependence of $\gamma_{\|}(I)$, is not yet known. It is tempting to attribute this dependence to a (temperatureinduced) shift in frequency detuning between the polarized cavity modes and the gain spectrum [7]. However, this explanation seems to be ruled out by our experiments. Apart from subtleties in the scalar or tensor nature of $\gamma_{\text {lin }}$, this explanation predicts that the mode closest to gain center lases and that the current dependence of $\gamma_{0}$ is proportional to the effective birefringence $\nu_{0}$. In practice, we find both switches from low-to-high and high-to-low frequencies, and we find hardly any correlation between the slope $d \gamma_{0} / d I$ [in figures like Fig. 9(b)] and $\nu_{0}$. An alternative explanation has not yet been found. The observation that the dominant polarization is always vertical before and horizontal after the switch indicates that the physical mechanism behind the polarization switch is linked to either the design layout of the array or to the orientation of the crystalline wafer.

The diffusion coefficient $D$ can be estimated from the real-time switching dynamics, which was found to depend critically on switching current. VCSELs that switch their polarization above 8-9 $\mathrm{mA}$ exhibit the hysteresis shown in Fig. 9; for switching at lower current, however, the dominant polarization was not stable all the time, but hopped between two quasistationary polarization states. The time it takes the VCSEL to actually switch was found to be very small and could hardly be resolved with our photodiode and oscilloscope; we estimate it to be just below 2 ns. On the other hand, the average dwell time in the two quasistationary states was very much larger. This average dwell time was found to depend strongly on switching current; in VCSELs that switch just below $8.5 \mathrm{~mA}$ it was about $1 \mathrm{~s}$, for switching around $7 \mathrm{~mA}$ it had dropped to (sub)microsecond. The reason for this rapid change is of course the exponential dependence of $\langle T\rangle$ on $\gamma_{\text {non }} / D$ in Eq. (20). As the observed hopping is driven by polarization noise, it can be used to get an estimate thereof [see Eq. (20) for the case of dominant linear birefringence]. At $I \approx 8.5 \mathrm{~mA}$ an average dwell time of about $1 \mathrm{~s}$ combines with a nonlinear dichroism $\gamma_{\mathrm{non}} \approx 1.1 \mathrm{~ns}^{-1}$ to 
give a diffusion coefficient of $D \approx 12 \mu \mathrm{s}^{-1}$.

Alternatively, the diffusion coefficient $D$ can be estimated from the absolute strength of the polarization fluctuations, as given by the ratio of power in the dominant polarization and the orthogonal polarization, in combination with the effective dichroism $\gamma_{0}$ [see Eq. (19) for case of dominant linear birefringence]. This power ratio can be obtained most reliably from optical spectra like Fig. 3, by integration over the lasing and nonlasing peak, but one can also use the frequency-integrated projection noise, as e.g. in Fig. 6, or even the polarization-resolved light-current characteristic of the laser (as long as the higher-order modes remain weak). We found these estimates to be mutually consistent within a factor 1.5; at a typical current of $8.5 \mathrm{~mA}$ they all yielded $P_{\text {nonlasing }} / P_{\text {lasing }} \approx 0.7-1.0 \%$. Combined with $\gamma_{0} \approx 1.1 \mathrm{~ns}^{-1}$ this then corresponds to $D \approx 8-11 \mu \mathrm{s}^{-1}$ being in reasonable agreement with the earlier estimate.

As a final step we deduce the cavity loss rate $\kappa$ from the value of $D$, using Eq. (5b). We therefore express the intracavity photon number $S$ in terms of the VCSEL output power as $P_{\text {out }}=2 h \nu \eta \kappa S$, where $\eta$ is the outcoupling efficiency through the top mirror. At $I=8.5 \mathrm{~mA}$ we had $D=8$ $-11 \mu \mathrm{s}^{-1}$ at an output power of $1.8 \mathrm{~mW}$. For an ideal fourlevel laser, where $n_{\mathrm{sp}}=\eta=1$, this would make the estimated cavity loss rate $\kappa \approx 200 \mathrm{~ns}^{-1}$. A more realistic estimate, based on $n_{\mathrm{sp}}=1.5$ and $\eta=0.3$, gives $\kappa \approx 300 \mathrm{~ns}^{-1}$.

\section{RESULTS FOR OTHER VCSELS}

In order to study the generic validity of our results we have repeated the experiments discussed above on another set of VCSELs, grown at the "Centre Suisse Electronique and Microtechnique', (former Paul Scherrer Institute) in Zürich, Switzerland. These were etched-post devices with a post diameter of $17 \mu \mathrm{m}$ (i.e., no proton implantation) that comprise three 8-nm-thick GaAs quantum wells in a $1-\lambda$ cavity. The lower and upper Bragg mirror contain 20 and 40.5 pairs of graded $\mathrm{AlAs}-\mathrm{Al}_{.18} \mathrm{Ga}_{.82} \mathrm{As}$ layers, respectively. The device that was singled out for further study had a threshold current of $I_{\mathrm{th}}=4.1 \mathrm{~mA}$, operated in the fundamental transverse mode up to $2 I_{\text {th }}$, and exhibited a polarization switch around $5.5 \mathrm{~mA}$, at an output power of $0.30 \mathrm{~mW}$.

Figure 10 shows the effective birefringence $\left|\nu_{0}\right|$ and dichroism $\left|\gamma_{0}\right| /(2 \pi)$ measured as a function of laser current. The behavior of this etched-post VCSEL is quite similar to that of the proton-implanted VCSEL in Fig. 9. Once more, we observed hysteresis; when the current is increased the VCSEL polarization switches from $y$ to $x$ at $I=5.65 \mathrm{~mA}$; when the current is decreased the VCSEL polarization lingers on in $x$ and switches back at $I=5.46 \mathrm{~mA}$. Again, the effective birefringence exhibits a jump due to the nonlinear birefringence [Fig. 10(a)] and again the switch coincides with a minimum in the measured dichroism as a function of current $\gamma_{0}(I)$ [see Fig. 10(b)]. By relating the jump in Fig. 10 (a) to the nonlinear redshift we find $\alpha \gamma_{\text {non }}$ $\approx \pi(5.13-4.68) \mathrm{ns}^{-1}=1.4(1) \mathrm{ns}^{-1}$. By relating the effective dichroism inside the hysteresis loop to nonlinear effects we find $\gamma_{\text {non }} \approx 2 \pi \times 0.132 \mathrm{~ns}^{-1}=0.83(6) \mathrm{ns}^{-1}$. Combining these two results yields $\alpha=1.7(2)$, which is relatively low, but not unrealistic for thin quantum wells [25]. As a detail, we note that the effective dichroism inside the hysteresis loop is

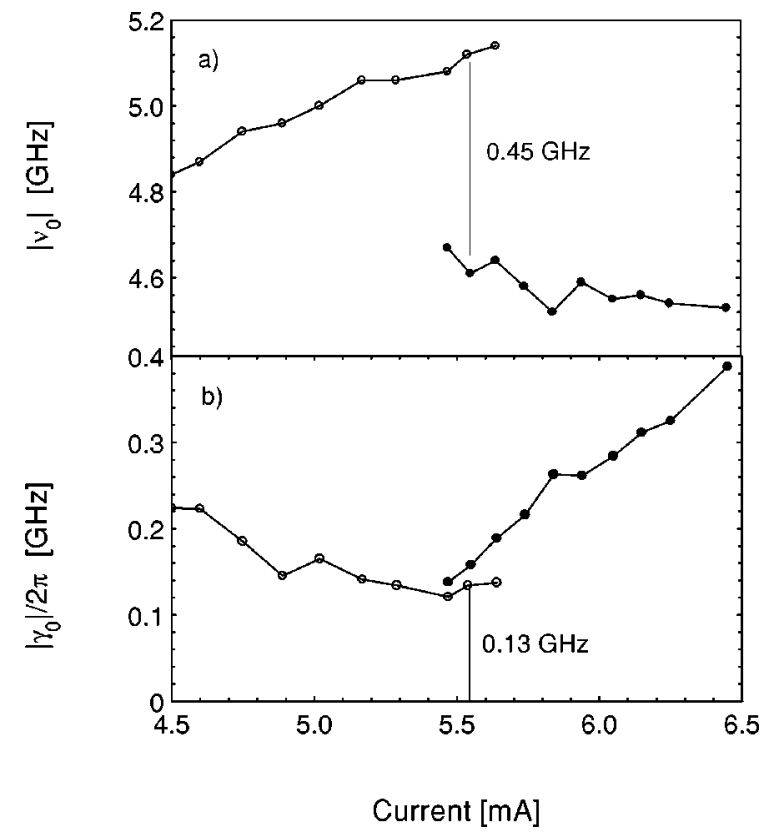

FIG. 10. The effective birefringence $\left|\nu_{0}\right|$ and dichroism $\left|\gamma_{0}\right|$ of the etched-post VCSEL as a function of current. Note the observed hysteresis and the jump in $\left|\nu_{0}\right|$ that occurs upon a polarization switch (around $I=5.5 \mathrm{~mA}$ ). From (b) we conclude that the polarization switch results from a current dependence dichroism, $\gamma_{0}(I)$.

asymmetric, $\gamma_{0}$ being larger after the polarization switch than before. The reason for this asymmetry is not yet known.

As a next step we tried to observe the effect of the nonlinear anisotropies in the polarization-resolved optical and intensity noise spectra. To increase our changes of success, and to facilitate the comparison with earlier results, we set the laser current at $I=5.55 \mathrm{~mA}$, i.e., inside the hysteresis loop, after the polarization switch. At this point, both $\nu_{0}$ and $\gamma_{0}$ are relatively small, so that both the magnitude of the nonlinear effects and the polarization fluctuations are optimized. In this situation the optical spectra showed the integrated power in the nonlasing peak to be $2.6 \%$ of that of the lasing peak. What is more important, these spectra also showed the presence of a four-wave-mixing peak at an intensity of $8.0(6) \times 10^{-4}$ of that of the nonlasing peak. When we combine this ratio with $\left|\nu_{0}\right|=4.68 \mathrm{GHz}$ in Eq. (8) we find $\sqrt{\alpha^{2}+1} \gamma_{\text {non }}=1.7(1) \mathrm{ns}^{-1}$, in good agreement with the earlier estimate based on the observed nonlinear redshift.

We also measured the polarization-resolved intensity noise. The fits to these spectra were quite good, although they were somewhat hindered by the presence of a lowfrequency relaxation-oscillation peak around $2.3 \mathrm{GHz}$. After the polarization switch the fluctuations in the polarization angle $\phi$ were measured to be smaller than in the ellipticity angle $\chi$, as expected for a VCSEL in which the lowfrequency mode dominates $\left(\nu_{0}=-4.68 \mathrm{GHz}\right)$. At the resonance frequency we measure $\left\langle|\phi(\omega)|^{2}\right\rangle^{1 / 2} /\left\langle|\chi(\omega)|^{2}\right\rangle^{1 / 2}$ $=0.92(2)$. Substitution of this ratio in Eq. (12) yields $\alpha \gamma_{\text {non }} \approx 2.4(6) \mathrm{ns}^{-1}$. This estimate is somewhat larger than the previous ones, but still falls within the error bars, which are relatively large due to the presence of relaxation oscillations. 
Finally we estimate the magnitude of the polarization noise from the observed power ratio $P_{\text {nonlasing }} / P_{\text {lasing }}$ $=2.6 \%$. Substitution of this ratio, and the fitted value of $\gamma_{0}=0.83 \mathrm{~ns}^{-1}$, into Eq. (19) yields a diffusion coefficient $D=22(3) \mu \mathrm{s}^{-1}$. Just as before, we now insert $D$, together with the output power of $0.3 \mathrm{~mW}$, into Eq. (5b), to obtain an estimated cavity loss rate $\kappa \approx 120 \mathrm{~ns}^{-1}$ for the ideal fourlevel laser and $\kappa \approx 220 \mathrm{~ns}^{-1}$ for the case $n_{\mathrm{sp}}=1.5$ and $\eta$ $=0.2$.

Comparing the etched-post VCSELs with the protonimplanted VCSELs we note that for both types of devices nonlinear effects were observable in three different ways, as (i) a nonlinear redshift and extra dichroism, (ii) a FWM peak in the optical spectrum, and (iii) a different magnitude of the projected polarization noise. Although the etched-post device switched at an output power that was only about $20 \%$ of that the proton-implanted devices, the observed nonlinear anisotropies were still sizeable $\left[\alpha \gamma_{\text {non }} \approx 1.5(2) \mathrm{ns}^{-1}\right.$, $\left.\gamma_{\text {non }} \approx 0.8 \mathrm{~ns}^{-1}\right]$ at about $50 \%$ of the values of the latter devices. The reason for this is that the cavity loss rate $\kappa$ of our etched-post device is relatively low (Bragg mirrors with more periods) so that a given output power corresponds to a relatively high internal field. Experimentally, this was also noticeable in the diffusion coefficient $D$, which at $D$ $\approx 22(3) \mu \mathrm{s}^{-1}$ for the etched-post device is only a factor of 2-3 larger than that of the proton-implanted device, despite the factor of 5 lower output power. To account for the difference in $\kappa$ it might be better to relate the degree of saturation to the quantity $\left(I / I_{\mathrm{th}}\right)-1$, which, around the polarization switch, was $\approx 0.35$ for our etched-post device (I $\left.=5.55 \mathrm{~mA} ; I_{\mathrm{th}}=4.1 \mathrm{~mA}\right)$ and $0.4-0.8$ for our protonimplanted devices $\left(I=7-9 \mathrm{~mA} ; I_{\mathrm{th}}=5.0 \mathrm{~mA}\right)$.

\section{SUMMARY AND CONCLUSIONS}

We have presented a general description of polarization fluctuations in VCSELs, allowing direct comparison with experiment. An overview of the model parameters is given in Table I. In total the model involves four anisotropies. The physical mechanism behind these anisotropies is not yet fully understood; we know how the linear birefringence arises from mechanical strain [27] and internal electrical fields [28], and how the nonlinear anisotropies result from the (eliminated) spin dynamics [6,11], but the origin, and in particular the experimental observation of a current dependence, of the linear dichroism $\gamma_{\text {lin }}(I)$ is still somewhat of a mystery.

In the experimental sections we have shown how the various parameters can be extracted from the experimental data. More specifically, the effective birefringence and effective dichroism appear as frequency splittings and widths in both optical and projected-intensity spectra. We gave three experimental demonstrations of the presence of nonlinear anisotropies. We have shown how they give rise to a four-wavemixing peak in the optical spectrum and to a nonuniformity in the projected polarization noise. Experimentally, both phenomena can be used to quantify the combined nonlinear anisotropies, but both are inversely proportional to (the square of) the linear birefringence so that the effects are measurable only for small to moderate birefringence. As a third demonstration of nonlinear effects we have shown how they give rise to a spectral redshift and excess width of the non- lasing peak as compared to the lasing peak. Experimentally, these measurements are ideal to separately determine the nonlinear birefringence and the nonlinear dichroism, but they only work for VCSELs that exhibit a polarization switch. Specifically, we have shown how in extreme cases, where the linear and nonlinear anisotropies are comparable, the concept of two polarization modes loses its meaning.

For a batch of proton-implanted VCSEL we have applied the three techniques mentioned above to obtain results that agreed within about $20 \%$. We have determined the nonlinear birefringence to be $\alpha \gamma_{\text {non }} \approx 3-4 \mathrm{~ns}^{-1}$ around $I=9 \mathrm{~mA}$ and $\mathrm{P}_{\text {out }}=1.9 \mathrm{~mW}$ and to be $\approx 2.5 \mathrm{~ns}^{-1}$ around $I=7 \mathrm{~mA}$ and $\mathrm{P}_{\text {out }}=1.3 \mathrm{~mW}$. The nonlinear dichroism was found to be a factor $\alpha \approx 3$ lower. We have also demonstrated to what extent the fluctuations in polarization direction $\phi$ and ellipticity $\chi$ are correlated. In general, we have shown how polarization fluctuations result from a balance between diffusion, due to polarization noise, and a restoring drift, due to dichroism. The diffusion coefficient $D$, and the related cavity loss rate $\kappa$ could thus be estimated from the relatively power in the nonlasing polarization and from the average hopping time in case of polarization switching. Repeating the measurements on a batch of etched-post VCSELs from a different supplier gave similar results. At lower current $(I=5.5 \mathrm{~mA})$ and lower output power $(P=0.3 \mathrm{~mW})$ we now found $\alpha \gamma_{\text {non }} \approx 1.5 \mathrm{~ns}^{-1}$, $\gamma_{\text {non }} \approx 0.8 \mathrm{~ns}^{-1}$, and $\alpha \approx 1.7$. Once more the three different measurements were in reasonable agreement. This shows that both the phenomena and the quoted numbers are rather general and not limited to a special type of VCSEL.

In conclusion, this work presents an experimental confirmation of the validity of the spin-eliminated model for the polarization behavior of a VCSEL. We have stressed that almost any practical VCSEL satisfies the condition for spin elimination. Also, most practical VCSELs satisfy additionally the condition of relatively strong linear birefringence which, in turn, greatly simplifies the analytic description. This being said, it remains very interesting, from a theoretical point of view, to study VCSELs which do not satisfy the condition for spin elimination. In particular, one would like to have a VCSEL with very large birefringence $\left(\omega_{\text {lin }}\right.$ $\left.>\gamma_{s} / \alpha\right)$ which exhibits a polarization switch; the switching behavior of this VCSEL should violate the framework exposed in the present paper. So far, we have not been able to find such a VCSEL among the many that we have studied.

\section{ACKNOWLEDGMENTS}

We acknowledge support from the "Stichting voor Fundamenteel Onderzoek der Materie (FOM),' from the European Union under the ESPRIT Project No. 20029 (ACQUIRE), and from TMR Network No. ERB4061 PL951021 (Microlasers and Cavity QED). We thank M. Moser and K. H. Gulden of the "Center Suisse Electronique and Microtechnique"' (formerly the Paul Scherrer Institute) in Zürich, Switzerland for kindly providing the etched-post VCSELs.

\section{APPENDIX}

In this appendix we will derive the steady-state polarization and linearized polarization rate equations for a laser 
where the linear birefringence and linear dichroism make an arbitrary angle $\beta$. For this case, the full rate equations, as found in $[6,13,14]$, are

$$
\begin{aligned}
2 \cos 2 \chi \frac{d \phi}{d t}= & -\omega_{\text {lin }} \sin 2 \chi \cos 2 \phi-\gamma_{\text {lin }} \sin 2(\phi-\beta) \\
& -2 \alpha \gamma_{\mathrm{non}} \sin 2 \chi \cos 2 \chi \\
2 \frac{d \chi}{d t}= & \omega_{\text {lin }} \sin 2 \phi-\gamma_{\text {lin }} \sin 2 \chi \cos 2(\phi-\beta) \\
& -2 \gamma_{\mathrm{non}} \sin 2 \chi \cos 2 \chi
\end{aligned}
$$

These equations are exact in the adiabatic limit; i.e., no assumptions have been made apart from the adiabatic elimination of the difference inversion. To remove the various sine and cosine functions we expand to first order in $\phi, \chi \ll 1$, assuming the intensity $I$, which codetermines $\gamma_{\text {non }}=\kappa I / \Gamma$, to be more or less constant (valid for operation reasonably far above threshold). The steady-state angles thus found are

$$
\begin{gathered}
\chi_{\mathrm{ss}} \approx \frac{\gamma_{\mathrm{lin}} \sin 2 \beta}{2\left(\omega_{\mathrm{lin}}+2 \alpha \gamma_{\mathrm{non}}\right)} \ll 1, \\
\phi_{\mathrm{ss}} \approx\left(\frac{\gamma_{\mathrm{lin}} \cos 2 \beta+2 \gamma_{\mathrm{non}}}{\omega_{\mathrm{lin}}}\right) \chi_{\mathrm{ss}} \ll 1 .
\end{gathered}
$$

Equation (A2a) is an extension to the nonlinear regime, of Eq. (18) in [9] that was derived from a linear coupled-mode theory. Note that this equation is asymmetric in (the sign of) $\omega_{\text {lin }}$; large ellipticity are most likely for negative $\omega_{\text {lin }}$, i.e., for the case where the low-frequency mode lases. For the case of dominant linear birefringence $\left(\omega_{\text {lin }} \gg \gamma_{\text {lin }}, \gamma_{\text {non }}\right)$ we also find $\phi_{\text {ss }} \ll \chi_{\text {ss }}[$ see Eq. (A2b)].

For $\chi_{\mathrm{ss}}, \phi_{\mathrm{ss}} \ll 1$ the linearized polarization rate equations, including noise, are

$$
\frac{d}{d t}\left(\begin{array}{c}
\phi-\phi_{\mathrm{ss}} \\
\chi-\chi_{\mathrm{ss}}
\end{array}\right)=\left(\begin{array}{cc}
-\gamma_{\|} & -\omega_{\mathrm{lin}}-2 \alpha \gamma_{\mathrm{non}} \\
\omega_{\mathrm{lin}} & -\gamma_{\|}-2 \gamma_{\mathrm{non}}
\end{array}\right)\left(\begin{array}{c}
\phi-\phi_{\mathrm{ss}} \\
\chi-\chi_{\mathrm{ss}}
\end{array}\right)+\left(\begin{array}{c}
f_{\phi} \\
f_{\chi}
\end{array}\right),
$$

where $\gamma_{\|} \equiv \gamma_{\text {lin }} \cos 2 \beta$, and where we have added the Langevin noise sources $f_{\phi}$ and $f_{\chi}$. We want to stress that, as these equations result from a linearization in the adiabatic approximation, they apply to all cases where $\phi, \chi, \chi_{\text {ss }} \ll 1$, including the very interesting cases where linear and nonlinear anisotropies are comparable in strength. Note that Eq. (A3) becomes identical to Eq. (6) in [11] and Eq. (1) in [2] for the case of aligned linear birefringence and dichroism.
[1] C. H. Henry, IEEE J. Quantum Electron. 18, 259 (1982).

[2] M. P. van Exter, A. Al-Remawi, and J. P. Woerdman, Phys. Rev. Lett. 80, 4875 (1998).

[3] M. San Miguel, Q. Feng, and J. V. Moloney, Phys. Rev. A 52, 1728 (1995).

[4] J. Martin-Regalado, F. Prati, M. San Miguel, and N. B. Abraham, IEEE J. Quantum Electron. 33, 765 (1997).

[5] M. Travagnin, M. P. van Exter, A. K. Jansen van Doorn, and J. P. Woerdman, Phys. Rev. A 54, 1647 (1996); 55, 4641(E) (1997).

[6] M. P. van Exter, R. F. M. Hendriks, and J. P. Woerdman, Phys. Rev. A 57, 2080 (1998).

[7] K. D. Choquette, R. P. Schneider, Jr., K. L. Lear, and R. E. Leibenguth, IEEE J. Sel. Top. Quantum Electron. 1, 661 (1995).

[8] J. Martin-Regalado, J. L. A. Chilla, J. J. Rocca, and P. Brusenbach, Appl. Phys. Lett. 70, 3350 (1997).

[9] A. K. Jansen van Doorn, M. P. van Exter, A. M. van der Lee, and J. P. Woerdman, Phys. Rev. A 55, 1473 (1997).

[10] H. van der Lem and D. Lenstra, Opt. Lett. 22, 1698 (1997).

[11] H. F. Hofmann and O. Hess, Quantum Semiclassic. Opt. 10, 87 (1998).

[12] M. Born and E. Wolf, Principles of Optics (Pergamon Press, Oxford, 1980).

[13] W. Van Haeringen, Phys. Rev. 158, 256 (1967).

[14] M. Travagnin, Phys. Rev. A 56, 4094 (1997).
[15] K. Petermann, Laser Diode Modulation and Noise (Kluwer, Dordrecht, 1988).

[16] M. P. van Exter, W. A. Hamel, J. P. Woerdman, and B. R. P. Zeijlmans, IEEE J. Quantum Electron. 28, 1470 (1992).

[17] D. V. Kuksenkov, H. Temkin, and S. Swirhun, Appl. Phys. Lett. 67, 2141 (1995).

[18] C. W. Gardiner, Handbook of Stochastic Methods (Springer, Berlin, 1985).

[19] S. Ciuchi, M. San Miguel, and N. B. Abraham, Phys. Rev. A 57, 3843 (1998).

[20] R. Roy, R. Short, J. Durnin, and L. Mandel, Phys. Rev. Lett. 45, 1486 (1980).

[21] D. Lenstra and S. Singh, Phys. Rev. A 28, 2318 (1983).

[22] M. B. Willemsen, M. P. van Exter, and J. P. Woerdman (unpublished).

[23] R. F. M. Hendriks, M. P. van Exter, J. P. Woerdman, K. H. Gulden, and M. Moser, IEEE J. Quantum Electron. 34, 1455 (1998).

[24] Vixel Corporation, 325 Interlocken Parkway, Broomfield, CO 80021, model PRI-LA-S-850-1x8-3S.

[25] M. Osinski and J. Buus, IEEE J. Quantum Electron. 23, 9 (1987).

[26] M. P. van Exter, M. B. Willemsen, and J. P. Woerdman (unpublished).

[27] A. K. Jansen van Doorn, M. P. van Exter, and J. P. Woerdman, Appl. Phys. Lett. 69, 1041 (1996).

[28] M. P. van Exter, A. K. Jansen van Doorn, and J. P. Woerdman, Phys. Rev. A 56, 845 (1997). 